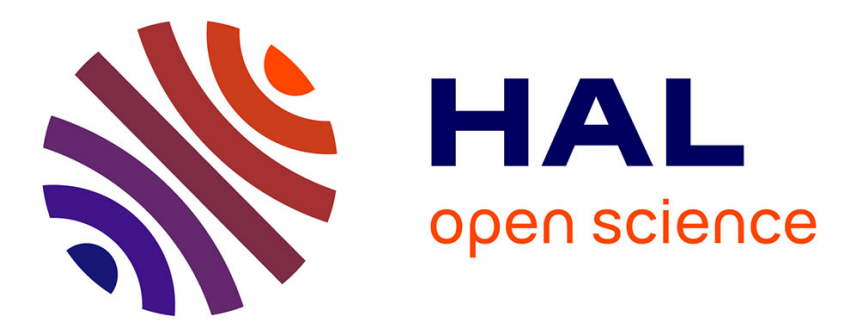

\title{
Characterization of perinatally born glutamatergic neurons of the mouse olfactory bulb based on NeuroD6 expression reveals their resistance to sensory deprivation
}

Alexandra Angelova, Jean-Claude Platel, Christophe Béclin, Harold Cremer, Nathalie Coré

\section{To cite this version:}

Alexandra Angelova, Jean-Claude Platel, Christophe Béclin, Harold Cremer, Nathalie Coré. Characterization of perinatally born glutamatergic neurons of the mouse olfactory bulb based on NeuroD6 expression reveals their resistance to sensory deprivation. Journal of Comparative Neurology, 2019, 527 (7), pp.1245-1260. 10.1002/cne.24621 . hal-02160586

\section{HAL Id: hal-02160586 \\ https://hal-amu.archives-ouvertes.fr/hal-02160586}

Submitted on 19 Jun 2019

HAL is a multi-disciplinary open access archive for the deposit and dissemination of scientific research documents, whether they are published or not. The documents may come from teaching and research institutions in France or abroad, or from public or private research centers.
L'archive ouverte pluridisciplinaire HAL, est destinée au dépôt et à la diffusion de documents scientifiques de niveau recherche, publiés ou non, émanant des établissements d'enseignement et de recherche français ou étrangers, des laboratoires publics ou privés. 
archives-ouvertes

\section{Characterization of perinatally born glutamatergic neurons of the mouse olfactory bulb based on NeuroD6 expression reveals their resistance to sensory deprivation} Alexandra Angelova, Jean-Claude Platel, Christophe Béclin, Harold Cremer, Nathalie Coré

\section{To cite this version:}

Alexandra Angelova, Jean-Claude Platel, Christophe Béclin, Harold Cremer, Nathalie Coré. Characterization of perinatally born glutamatergic neurons of the mouse olfactory bulb based on NeuroD6 expression reveals their resistance to sensory deprivation. Journal of Comparative Neurology, John Wiley

Sons, 2019, 527 (7), pp.1245-1260. 10.1002/cne.24621 . hal-02160586

\section{HAL Id: hal-02160586 \\ https://hal-amu.archives-ouvertes.fr/hal-02160586}

Submitted on 19 Jun 2019

HAL is a multi-disciplinary open access archive for the deposit and dissemination of scientific research documents, whether they are published or not. The documents may come from teaching and research institutions in France or abroad, or from public or private research centers.
L'archive ouverte pluridisciplinaire $\mathbf{H A L}$, est destinée au dépôt et à la diffusion de documents scientifiques de niveau recherche, publiés ou non, émanant des établissements d'enseignement et de recherche français ou étrangers, des laboratoires publics ou privés. 


\title{
Characterization of perinatally born glutamatergic neurons of the mouse olfactory bulb based on NeuroD6 expression reveals their resistance to sensory deprivation
}

\author{
Alexandra Angelova | Jean-Claude Platel | Christophe Béclin | Harold Cremer | \\ Nathalie Coré $\mathbb{0}$
}

Aix Marseille Univ, CNRS UMR 7288, Developmental Biology Institute of Marseille (IBDM), Parc scientifique de Luminy, Marseille, France

\section{Correspondence}

Harold Cremer, Aix Marseille Univ, CNRS UMR 7288, Developmental Biology Institute of Marseille (IBDM), Parc scientifique de Luminy, 13009, Marseille, France.

Email: harold.cremer@univ-amu.fr

Funding information

Agence Nationale de la Recherche, Grant/ Award Numbers: ANR-10-INBS-04, ANR13-BSV4-0013; Fondation A*MIDEX- Biotrail program; Association France Parkinson; Fondation de France, Grant/Award Number: FDF70959; Fondation pour la Recherche Médicale, Grant/Award Numbers: FDT20160435597, ING20150532361

\begin{abstract}
During postnatal olfactory bulb (OB) neurogenesis, predetermined stem cells residing in the ventricular-subventricular zone continuously generate progenitors that migrate in the rostral migratory stream and integrate into the OB. Although the vast majority of these postnatally generated interneurons are inhibitory, a sub-fraction represents glutamatergic neurons that integrate into the superficial glomerular layer. In the present work, we demonstrate that the bHLH transcription factor NeuroD6 is specifically and transitorily expressed in the dorsal neurogenic lineage that generates glutamatergic juxtaglomerular cells (JGCs) for the OB. Using lineage tracing combined with whole brain clearing, we provide new insight into timing of generation, morphology, and connectivity of glutamatergic JGCs. Specifically, we show that all glutamatergic JGCs send complex axons with varying projection patterns into different layers of the OB. Moreover, we find that, contrary to GABAergic OB interneurons, glutamatergic JGCs survive under sensory deprivation, indicating that inhibitory and excitatory populations are differentially susceptible to environmental stimulation.
\end{abstract}

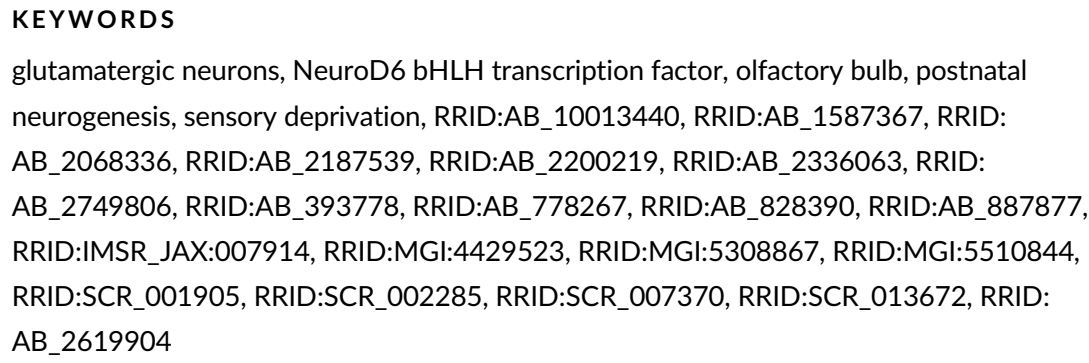

\section{1 | INTRODUCTION}

Postnatal and adult stem cell populations lining the lateral ventricles permanently generate neuronal precursors that migrate into the olfactory bulb $(\mathrm{OB})$ where they integrate into the granule and glomerular layers. The vast majority of these postnatal- and adult-born interneurons are inhibitory, using GABA and in part additionally dopamine as their neurotransmitters (Parrish-Aungst, Shipley, Erdelyi, Szabo, \& Puche, 2007). However, more recent work demonstrated that also excitatory glutamatergic neurons are produced during postnatal and in some cases adult stages (Brill et al., 2009; Winpenny et al., 2011). In addition to such heterogeneity at the neurotransmitter level, OB neurons can be sub-grouped by the expression of specific markers like calretinin (CaIR) or calbindin (CalB), their final position in the OB and their connectivity (Fiorelli, Azim, Fischer, \& Raineteau, 2015; Weinandy, Ninkovic, \& Gotz, 2011; Whitman \& Greer, 2009).

This diversity among $\mathrm{OB}$ interneurons reflects the regionalization of neural stem cell (NSC) pools around the lateral ventricle. Indeed, the position of NSCs in the ventricular-subventricular zone (V-SVZ) determines the above-described phenotypic features of the resulting 
interneurons (Merkle, Mirzadeh, \& Alvarez-Buylla, 2007). For example, purely GABAergic granule neurons are generated from NSCs located in the lateral subventricular zone. In contrast, CalB-positive neurons are derived from the ventro-lateral progenitor domain, whereas CalR-positive and dopaminergic ( $\mathrm{TH}+$ ) juxtaglomerular cells (JGC) are generated in medial and dorsal domains of the ventricular wall (Fuentealba et al., 2015).

Over the past decades, the generation, morphology, and function of postnatal and adult born inhibitory $O B$ interneurons have been extensively characterized (Whitman \& Greer, 2007). However, although there is substantial data concerning the structure and physiology of OB excitatory JGCs in general (Antal, Eyre, Finklea, \& Nusser, 2006; Aungst et al., 2003; Hayar, Karnup, Ennis, \& Shipley, 2004a), information concerning specifically the postnatal- and adult-born fraction is far more fragmentary. Lineage analyses based on the expression of the transcription factor Neurogenin2 (Neurog2) (Winpenny et al., 2011) provided insight into the sequence of generation and basic morphology/connectivity of these cells. Indeed, they are generated from the dorsal wall of the lateral ventricles and integrate after their migration into juxtaglomerular positions of the OB. Two major classes of Neurog2-derived JGCs have been defined so far. First, external tufted cells that extend complex primary dendrites inside a single glomerulus. Second, neurons with more extended dendritic arborizations were categorized as short axon cells (Winpenny et al., 2011). However, timing of their generation and the respective proportion that these two sub-populations represent in the postnatal $\mathrm{OB}$ is not well characterized. Also, although glutamatergic neurons in general have been shown to bear axons (Hayar, Karnup, Ennis, et al., 2004), specific information about the postnatally generated fraction is sparse.

A striking feature of the $O B$ concerns its sensitivity to sensory deprivation, making it a model of choice to study the impact of experience in the generation, maintenance, and renewal of brain circuitry Indeed, a considerable number of studies, using naris closure as an experimental paradigm, demonstrated a size reduction of the $\mathrm{OB}$ associated with cell loss of defined subsets of OB inhibitory interneurons including the tyrosine hydroxylase $(\mathrm{TH})$ expressing and the CalR and CalB positive subtypes (Bastien-Dionne, David, Parent, \& Saghatelyan, 2010; Bovetti, Veyrac, Peretto, Fasolo, \& De Marchis, 2009; Kato et al., 2012; Parrish-Aungst et al., 2011; Sawada et al., 2011). Information about the behavior of glutamatergic OB neurons under sensory deprivation is so far not available despite the key role of glutamatergic neurotransmission in olfactory processing.

We show here that the bHLH transcription factor NeuroD6 is specifically and transitorily expressed in the dorsal neurogenic lineage that generates glutamatergic JGCs for the olfactory bulb. We find that ND6 ${ }^{\text {CreERT2 }}$ mice allow the analysis of this cell type at high temporal and spatial resolution. Using this tool, we further characterize the timing of their generation and combine it with two-photon imaging of cleared brain to provide new insight of their morphology and connectivity. Finally, we combine ND6 lineage tracing with two-photon in vivo imaging to demonstrate that glutamatergic $O B$ neurons, unlike the inhibitory populations, survive even under conditions of sensory deprivation.

\section{2 | MATERIALS AND METHODS}

\section{1 | Animals}

In this study, NeuroD6 ${ }^{\mathrm{Cre}}$ (ND6 ${ }^{\mathrm{Cre}}$ ) knock-in mice (Goebbels et al. 2006), NeuroD6 ${ }^{\text {CreERT2 (ND6 }}{ }^{\text {CreERT2) }}{ }^{2}$ knock-in mice (Agarwal et al., 2012), Rosa26 ${ }^{\text {tdTomato }}$ reporter mice (Ai14, Jackson Laboratories, RRID:IMSR_JAX:007914), and GAD67 ${ }^{\text {GFP }}$ knock-in mice (Tamamaki et al., 2003) (RRID:MGI:5510844) of both sexes were used. Mouse lines were kept on a pure C57BL/6 genetic background unless they were crossed to Rosa $26^{\text {tdTomato }}$ reporter mice, which were maintained on an outbred CD 1 (Charles-River, Lyon, France) background. Mice were kept on a $12 \mathrm{hr}$ day/night cycle and had access to food and water ad libidum. All animal experiments were carried out in accordance to the European Communities Council Directive 2010/63/EU and approved by French ethical committees (Comité d'Ethique pour l'expérimentation animale no. 14; permission numbers: 00967.03; 2017112111116881v2)

\section{2 | Tamoxifen induction of Cre-mediated recombination}

For induction of Cre mouse lines, tamoxifen (TAM, T5648-1G, SIGMA) was injected subcutaneously for pups and intraperitoneally for juvenile and adult mice, respectively, at a dose of $100 \mathrm{mg} / \mathrm{kg}$. Typically, neonatal (PO-P6) ND6 ${ }^{\text {CreERT2 }}$ mice were injected for 2 days with one subcutaneous TAM injection/day. ND6 ${ }^{\text {CreERT2 }}$ mice at P15 and P45 were injected three and five times with TAM respectively, using 1 injection/day.

For intraventricular injection, the metabolically active component (Z)4-hydroxytamoxifen (4OH-TAM) was used. 4OH-TAM powder ( $\mathrm{H} 7904$ SIGMA) was dissolved in $96 \% \mathrm{EtOH}$ to obtain a $20 \mathrm{mM}$ stock solution. Typically, $2 \mu \mathrm{L}$ of $500 \mu \mathrm{M} 4 \mathrm{OH}$-TAM in sterile phosphate-buffered saline (PBS) were injected into neonatal (PO or P1) ND6 ${ }^{\text {CreERT2 }}$ mice.

\section{3 | Postnatal electroporation}

Postnatal electroporation was performed as described previously (Boutin, Diestel, Desoeuvre, Tiveron, \& Cremer, 2008). The pCAG Cre and pCAG-EGFP plasmids (Morin, Jaouen, \& Durbec, 2007) were purified using NucleoBond Xtra Maxiprep DNA extraction kit (Macherey-Nagel) following the manufacturer's protocol and resuspended in sterile PBS. Briefly, neonatal pups (PO-P1) were anesthetized by hypothermia. Approximately $2 \mu \mathrm{L}$ of $5 \mu \mathrm{g} / \mu \mathrm{L}$ plasmid DNA combined with $0.1 \%$ Fast Green were injected into the lateral ventricle by expiratory pressure using an aspirator tube assembly (Drummond) connected to a pulled glass capillary (30-0057, Harvard Apparatus). For electroporation, injected pups were subjected to $5 \times 95 \mathrm{~V}$ electrical pulses ( $50 \mathrm{~ms}$, separated by $950 \mathrm{~ms}$ intervals) using an CUY21 edit device (Nepagene, Chiba, Japan) and $10 \mathrm{~mm}$ tweezer electrodes (CUY650P10, Nepagene) coated with conductive gel (Control Graphique Medical, France). Orientation of the electrodes determined whether the dorsal or lateral portion of the V-SVZ was transfected. Pups were then reanimated in a $37^{\circ} \mathrm{C}$ incubator before returned to the mother. 


\subsection{Quantitative real-time polymerase chain reaction}

RNA extraction was performed using RNeasy Mini Kit (Qiagen) following the manufacturer's protocol. cDNAs were produced using Superscript III Reverse Transcriptase (Thermo Fisher Scientific). Quantitative polymerase chain reactions (QPCRs) were performed on a Bio-Rad CFX system using SYBR-GreenER qPCR Super-Mix (Thermo Fisher Scientific), with $\beta$-actin as a reference gene. Primers used for mRNA detection were: $\beta$-actin-FOR_CTAAGGCCAACCGTGAAAAG and $\beta$-actin-REV_ACCAGAGGCATACAGGGACA; ND6-FOR_GTTGATG CATGAATGCTGGT and ND6-REV_GTGACATTGATGCCAACTGC.

\section{5 | Primary antibody characterization}

The anti-Ankyrin $G$ antibody is used to label axonal initial segment (AIS) of neurons. This antibody specifically stains AIS of neurons in the mouse olfactory bulb similarly as it was described in a previous study (Kosaka \& Kosaka, 2011).

With the anti-CalR and anti-CalB antibodies, we stained two specific sub-populations of mature neurons within the glomerular layer of the olfactory bulb with a similar pattern as obtained in previous studies (de Chevigny et al., 2012; Tiveron et al., 2017).

The anti-Ki67 antibody recognizes a nuclear antigen exclusively expressed in proliferating cells. We observed extensive nuclear staining in the V-SVZ of the forebrain where proliferating progenitors reside. The antibody stained very few cells in brain area such as the corpus callosum or the olfactory bulb where few cells are supposed to divide.

The anti-Pax6 antibody labeled the nucleus of progenitor cells expressing the transcription factor Pax6 within the ventricular zone from the dorsal aspect of the lateral ventricle. This pattern is similar as it was observed in previous studies (de Chevigny et al., 2012).

The anti-RFP antibodies were used to amplify the signal emitted by tdTomato fluorescent protein, derivative of DsRed. This antibody only labeled cells that were recombined in the forebrain of ND6-Cre mice, with a similar pattern that was described for these mice (Agarwal et al., 2012; Goebbels et al., 2006).

The anti-Tbr1 and anti-Tbr2 antibodies specifically stained the nucleus of mature neurons expressing the transcription factors Tbr1 and Tbr2, in the glomerular layer of the olfactory bulb and progenitor cells in the subventricular zone from the dorsal aspect of the lateral ventricle, similarly as it was obtained in previous studies (Brill et al., 2009; Winpenny et al., 2011).

The anti-TH antibody recognized the $\mathrm{TH}$ which is specifically expressed in dopaminergic neurons. This antibody stained a subpopulation of mature neurons in the glomerular layer of the olfactory bulb in a pattern similar that has been observed in previous studies (de Chevigny et al., 2012; Qin, Ware, Waclaw, \& Campbell, 2017; Tiveron et al., 2017).

The anti-Vglut1 and anti-Vglut2 antibodies recognized vesicular glutamate transporters and revealed the nerve terminals of glutamatergic neurons in the olfactory bulb. We observed punctate staining in all layers where glutamatergic neurons project, including the mitral cell layer, the external plexiform layer, and the glomerular layer. These staining were similarly obtained in previous works (Brill et al., 2009; Roybon et al., 2015; Winpenny et al., 2011).

\section{6 | Immunohistochemistry}

For histological analysis, mice were intracardially perfused with $4 \%$ paraformaldehyde (PFA) using a peristaltic pump. Brains were subsequently dissected and postfixed in $4 \%$ PFA overnight at $4{ }^{\circ} \mathrm{C}$. The next day, brains were either subjected to cryoprotection in $30 \%$ sucrose or placed in PBS azide $0.01 \%$ for long-term storage at $4^{\circ} \mathrm{C}$. Typically, brains were sectioned in $50 \mu \mathrm{m}$ slices using a microtome (Microm, HM 450).

Standard immunostaining protocols were used, unless stated otherwise. Briefly, coronal free floating sections were rinsed in PBS and, if necessary, antigen retrieval was performed using a sodium citrate buffer (10 mM sodium citrate, 0.05\% Tween20, $\mathrm{pH}$ 6.0) for $20 \mathrm{~min}$ at $90^{\circ} \mathrm{C}$. Then, brain sections were incubated in blocking buffer $(10 \%$ fetal bovine serum [FBS], 0.3\% Triton X-100 in PBS) for $1 \mathrm{hr}$. Subsequently, sections were incubated in primary antibody solution $(5 \%$ FBS, $0.1 \%$ Triton X-100 in PBS [PBST], and primary antibody [listed in Table 1]) overnight at $4^{\circ} \mathrm{C}$. The following day, sections were rinsed three times in PBS and incubated with species-appropriate secondary antibody in PBST for $2 \mathrm{hr}$ at RT using gentle rocking. Alexa Fluorconjugated secondary antibodies were from Jackson ImmunoResearch. Nuclear counterstain HOECHST (Invitrogen, 1:2000) was added before sections were washed in PBS and mounted on glass slides using Mowiol as a mounting medium.

Images were acquired with laser scanning confocal microscopes (LSM780 or LSM880, Zeiss, Germany) using Plan-Apochromat 20x/0.8 NA and oil-immersion Plan-Apochromat 40x/1.4 NA objectives. Images were acquired with Zen software (Zeiss, RRID:SCR_013672), and processing was performed using Fiji software (RRID:SCR_002285, (Schindelin et al., 2012)).

\section{7 | Tissue clearing and lightsheet microscopy}

For whole brain clearing, the advanced Cubic protocol (Susaki et al., 2015) was used. Briefly, brains were incubated in Cubic1 solution ( $250 \mathrm{~g} / \mathrm{L}$ urea, $250 \mathrm{~g} / \mathrm{L}$ Quadrol, $150 \mathrm{~g} / \mathrm{L}$ Triton X-100) until they appeared transparent. For neonatal brains, this was typically 2-3 days, whereas adult brains incubated for 7-10 days. For subsequent immunostaining, brains were washed in PBS for 1-2 hr and incubated in blocking solution (5\% FBS, $0.1 \%$ Triton X-100, $0.1 \%$ Tween-20, and $0.01 \%$ sodium azide, in PBS) for 1 day. Then, brains were submerged in $2.5 \mathrm{~mL}$ blocking solution containing the primary antibody for 4 days. Antibody concentration was sometimes doubled as compared to classical immunostaining protocols to obtain a better signal-to-noise ratio. The samples were then washed 2-3 times for 1 day with washing solution (0.01\% Tween-20, $0.01 \%$ sodium azide, in PBS) and subsequently incubated for 3 days in $2.5 \mathrm{~mL}$ washing solution containing the Alexa Fluor-conjugated secondary antibodies and TOPRO-3 (1/1000) nuclear stain. Finally, the samples were washed again and incubated in Cubic1 for $2 \mathrm{hr}$, then in Cubic2 ( $25 \mathrm{~g} / \mathrm{L}$ urea, $50 \mathrm{~g} / \mathrm{L}$ sucrose, $10 \mathrm{~g} / \mathrm{L}$ triethanolamine) for $1-2$ days before imaging. All procedures were performed in a water bath at $37^{\circ} \mathrm{C}$ under gentle agitation. 
TABLE 1 Primary antibodies used in this study

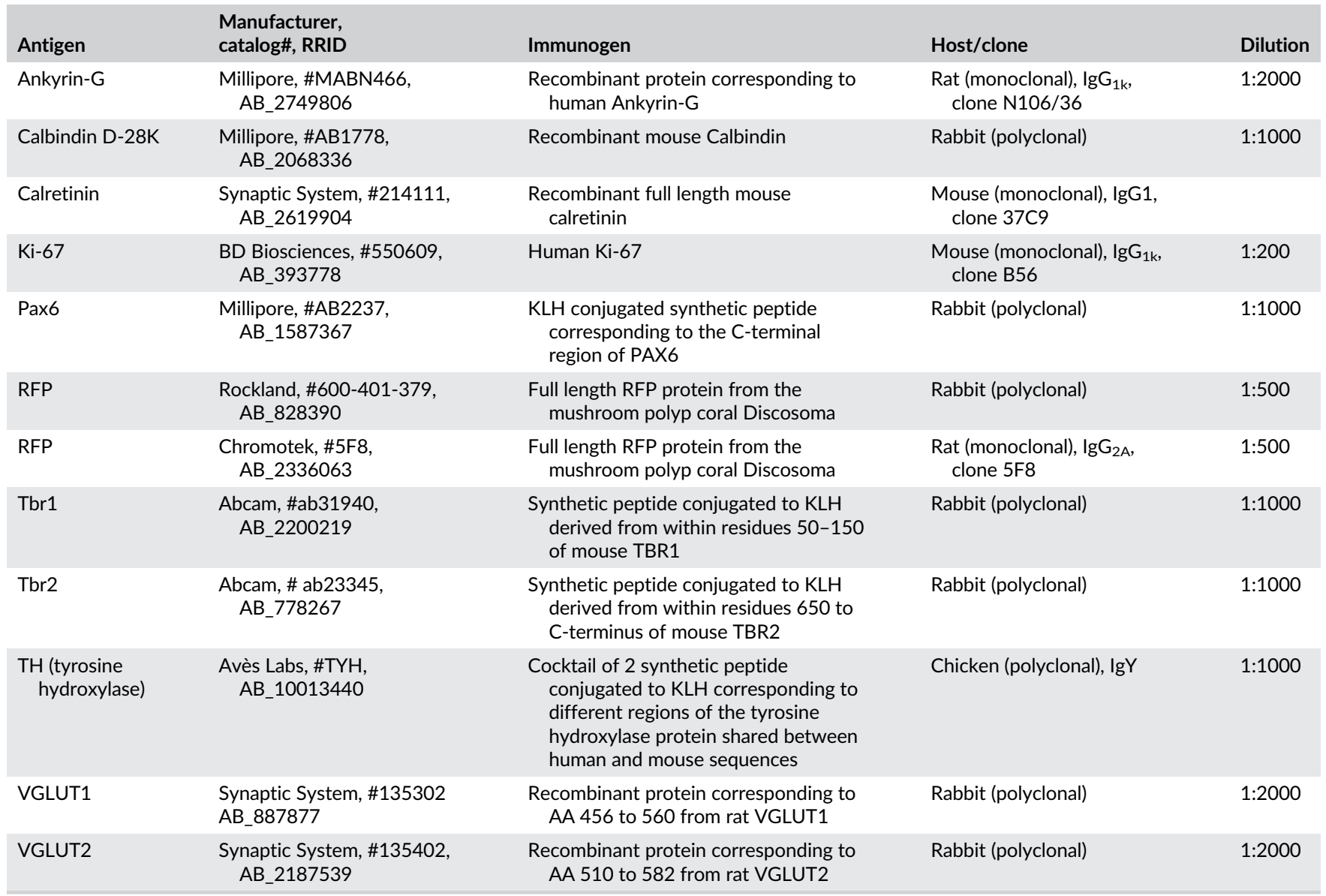

Whole tissue 3-D images were acquired using a Lightsheet Z.1 microscope (Zeiss, Germany) equipped with a 5x/0.16 NA objective (RI 1.45). The sample was glued caudally to the sample holder which was subsequently submerged in a chamber containing Cubic2 solution. Images were processed using ImageJ (NIH, https://imagej.nih. gov/ij/, RRID:SCR_003070) and Imaris (Bitplane, Zurich, Switzerland, RRID:SCR_007370) softwares.

For reconstruction of axonal processes, neonatal ND6 ${ }^{\text {CreERT2/ }}$ tdTom mice were induced by intraventricular injection of $4 \mathrm{OH}-\mathrm{TAM}$ to obtain very sparse labeling which allowed for following single processes. Mice were sacrificed at 6 wpi (weeks post induction), and brains subjected to the Cubic tissue clearing protocol, with the difference that brains were imaged in Cubic1 instead of Cubic2 solution. Therefore, the incubation step with Cubic2 was skipped. Images were acquired with a 7MP two-photon microscope (Zeiss, Germany) using a 20x/1.0 NA objective. Neurons were traced using Simple Neurite Tracer plugin of ImageJ (NIH). Reconstructions were represented using NeuTube (Feng, Zhao, \& Kim, 2015) software.

\section{8 | Stereotaxic injection and retrograde tracing}

For retrograde tracing of axonal processes, 6 weeks old ND6 $6^{\text {Cre/tdTom }}$ mice were anesthetized with intraperitoneal injection (i.p.) of ketamine/xylazine (125 and $12.5 \mathrm{mg} / \mathrm{kg}$, respectively). Before surgery started, mice received subcutaneous injections of carprofen $(5 \mathrm{mg} / \mathrm{kg}$ ), dexamethasone $(0.2 \mathrm{mg} / \mathrm{kg})$, and buprenorphine $(0.3 \mathrm{mg} / \mathrm{kg})$. Mice were placed in a stereotaxic apparatus (Harvard Apparatus); the skin over both OBs was incised with a scalpel and gently moved aside for better access to the skull. Injection point was identified (AP: $+5 \mathrm{~mm}$, ML: +1.1 mm, DV: 200-300 $\mu \mathrm{m}$ ) using Bregma coordinates, and a small hole was drilled into the skull to allow subsequent injection. Mice received $500 \mathrm{~nL}$ of $2 \mathrm{mg} / \mathrm{mL}$ CTB (cholera toxin subunit B, Thermo Fisher Scientific) conjugated with Alexa Fluor 488 using a Hamilton syringe. One week after CTB injections, mice were intracardially perfused with $4 \%$ PFA.

\subsection{Thinned skull technique for chronic in vivo observation of neuronal survival}

To visualize glutamatergic $\mathrm{OB}$ neurons and follow their survival, neonatal

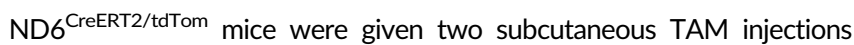
(one injection per day). To visualize GABAergic neurons, pCAG-Cre plasmid was dorsally electroporated into neonatal Rosa2 $6^{\text {tdTom }}$ mice. Mice were subjected to surgery when 4 weeks old. The thinned-skull technique (Shih, Mateo, Drew, Tsai, \& Kleinfeld, 2012; Yang, Pan, Parkhurst, Grutzendler, \& Gan, 2010) was used to allow in vivo observation of neuronal populations over time. Briefly, mice were anesthetized with ketamine/xylazine. Additional subcutaneous injections of carprofen $(5 \mathrm{mg} / \mathrm{kg})$, dexamethasone $(0.2 \mathrm{mg} / \mathrm{kg})$, and buprenorphine $(0.3 \mathrm{mg} / \mathrm{kg})$ were performed before surgery. Skin over both OBs was incised with a surgical scalpel blade and gently pushed aside to expose the skull. Surface of the skull was scrubbed with the scalpel and cleaned with $10 \%$ 
(a)

(b)
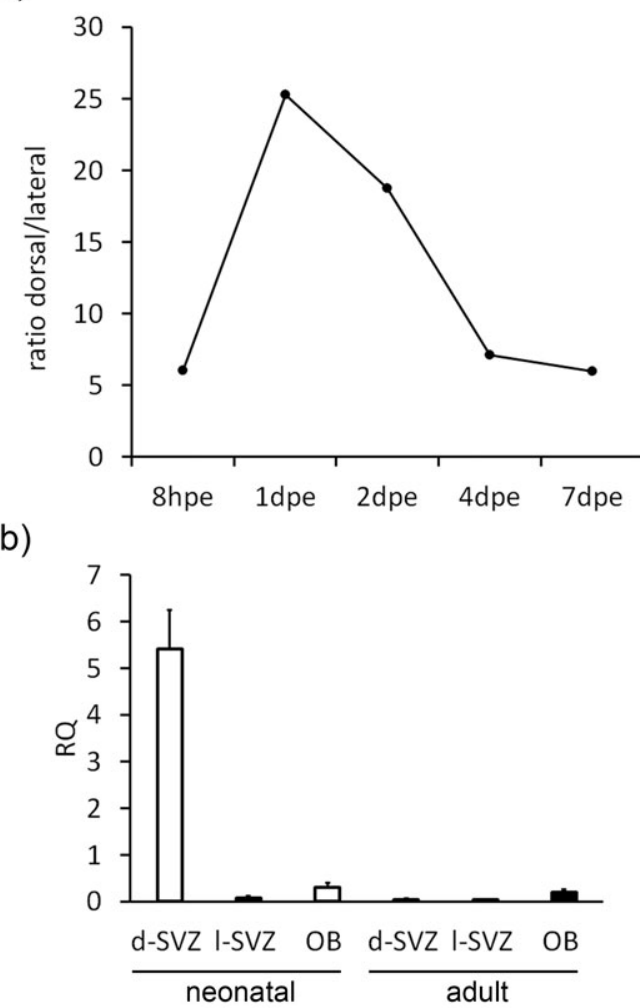

(c)

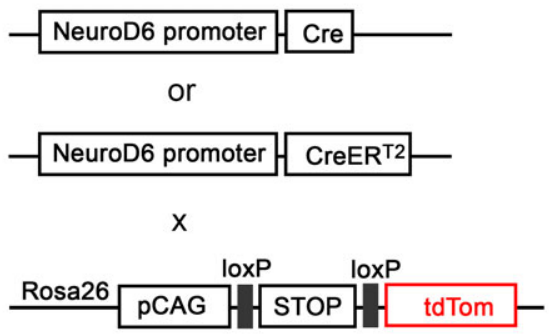

(d)

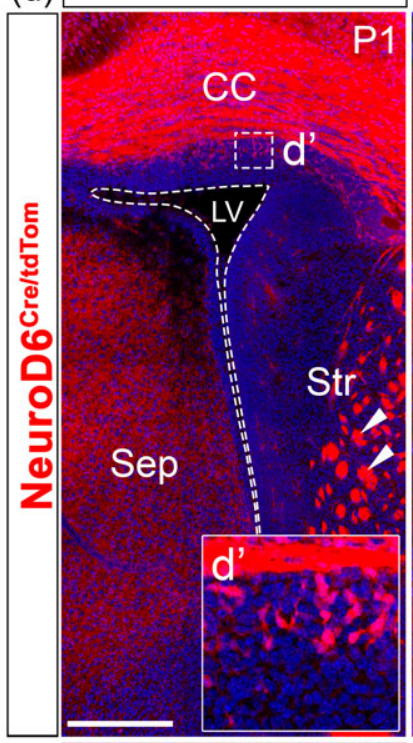

(e) $\mathrm{OB}$

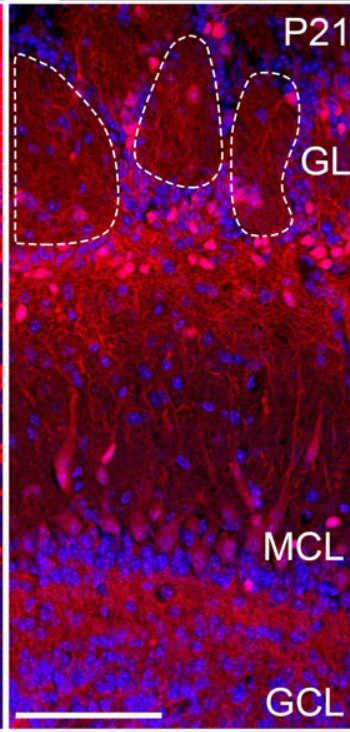

(f)
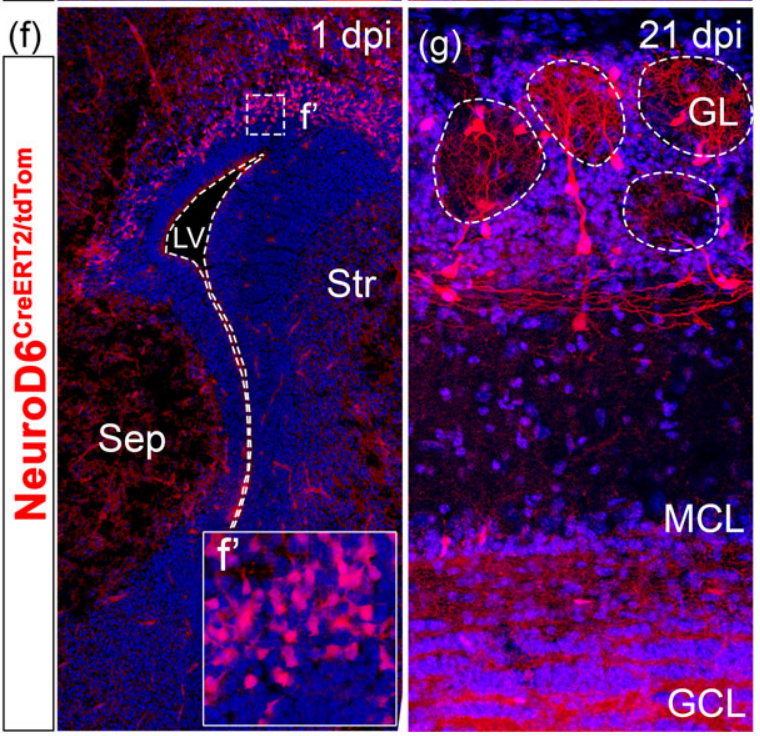

FIGURE 1 NeuroD6 marks a progenitor pool situated in the dorsal V-SVZ. (a) Relative expression ratio of NeuroD6 (ND6) in sorted cells of dorsal versus lateral neurogenic lineages at different time points of the neurogenic progression. Data originates from micro-array-based screen published by (Tiveron et al., 2017). Ratios show that dorsal expression is predominant. (b) Confirmation of ND6 expression data with quantitative RT-PCR on micro-dissected tissue originating from dorsal (d-SVZ) and lateral (I-SVZ) V-SVZ, as well as OB tissue from neonatal and adult animals, respectively. Expression levels are highest in d-SVZ sample. RQ: Relative quantification. (c) Schematic representation of Cre recombinase knock-in in ND6 ${ }^{\mathrm{Cre}}$ and ND6 ${ }^{\mathrm{CreERT} 2}$ lines crossed to Rosa26 $6^{\text {tdTomato }}$ (tdTom) reporter line. (d) V-SVZ of neonatal (P1) ND6 ${ }^{\text {Cre/tdTom }}$ mice. Note that recombined tdTomato (tdTom) cells are present in the dorsal sub-portion of the V-SVZ (d'). (e) OB of juvenile (P21) ND6 $6^{\text {Cre/tdTom }}$ mice. Note that recombined cells are present in the mitral cell layer (MCL) and in the glomerular layer (GL). (f) V-SVZ of induced (1 dpi) neonatal ND6 ${ }^{\text {CreERT2/tdTom }}$ mice after induction with tamoxifen (Tam) at PO. Note that again recombined cells are present in the dorsal sub-portion of the V-SVZ ( $f^{\prime}$ ). (g) OB of induced ( $21 \mathrm{dpi}$ ) juvenile ND6 ${ }^{\text {Cre/tdTom }}$ mice after TAM induction at PO. Note that recombined cells are almost absent from the MCL and less cells are present in the GL as compared to (e). Nuclei are stained in blue with Hoechst. White contour lines in the $\mathrm{OB}(\mathrm{e}, \mathrm{g})$ delineate glomeruli. hpe: hours post electroporation, dpe: days post electroporation, dpi: days post induction. CC: corpus callosum, GCL: granule cell layer, LV: lateral ventricle, OB: olfactory bulb, Sep: Septum, Str: Striatum, V-SVZ: ventricular-subventricular zone. Scale bars: (d) $200 \mu \mathrm{m}$; (e) $100 \mu \mathrm{m}$ [Color figure can be viewed at wileyonlinelibrary.com]

citric acid to ensure adhesion. A custom-made metal bar was then fixed caudally to the OBs using first superglue and then dental cement (SuperBond $C \& B$ ). Subsequently, the mouse was attached to a stereotaxic apparatus, using the metal bar. The skull was then gently thinned using a surgical scalpel blade until a thickness of approximately $15 \mu \mathrm{m}$ was reached over the desired area. During this procedure, great care was taken not to exert excessive pressure and to avoid bleeding or drying of the skull. Once the desired thickness was reached, a $3 \mathrm{~mm}$ cover slip was placed with the aid of a drop of superglue over the thinned area and sealed with dental cement. The preparation was left to dry for 1-2 min before imaging. If desired, unilateral nasal occlusion was performed (see section for details). Fluorescently labeled neurons of alive mice were then imaged with a 7MP two-photon microscope (Zeiss, Germany) using a water-immersion $20 \times / 1.0$ NA objective. Images were acquired at 


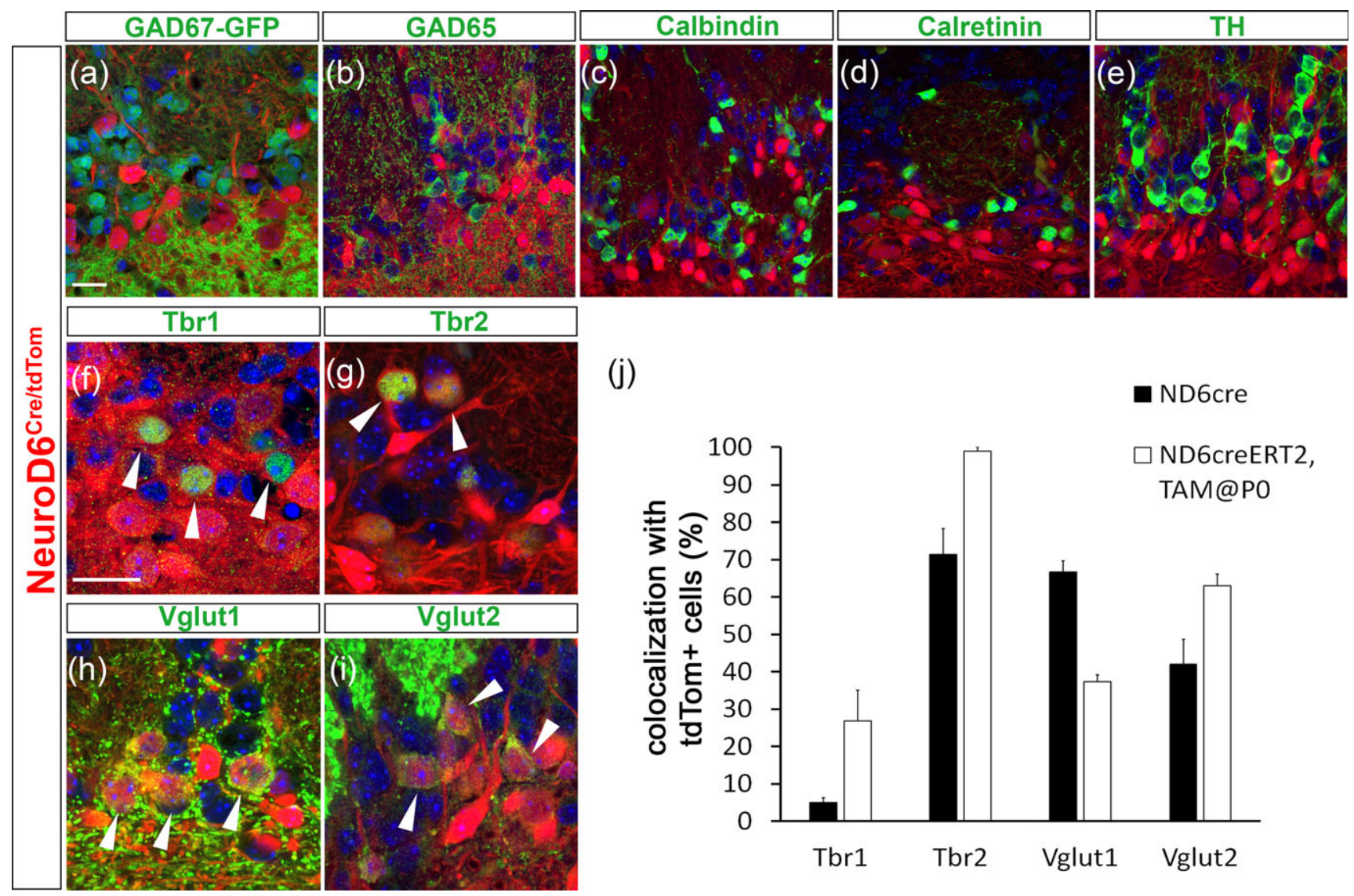

FIGURE 2 ND6-derived OB neurons display a glutamatergic phenotype. No co-localization between known GABAergic OB markers (in green) GAD67-GFP (a), GAD65 (b), calbindin (c), calretinin (d), tyrosin hydroxylase (TH, e), and tdTom+ cells in constitutively Cre-expressing line (ND6 ${ }^{\mathrm{Cre}}$ ). tdTom + cells co-localize (arrow heads) with glutamatergic markers (in green) Tbr1 (f), Tbr2 (g), Vglut1 (h), and Vglut2 (i).

(j) Quantification of co-localization of glutamatergic markers with tdTom+ neurons in both ND6 mouse lines. Proportion of double positive cells varies dependent on the labeling strategy (constitutively expressed ND6 ${ }^{\text {Cre }}$ which marks all glutamatergic neurons versus inducible ND6 ${ }^{\text {CreERT2 }}$ line which marks a subpopulation of neurons born around PO). Scale bars: (a, f) $20 \mu \mathrm{m}$. Error bars indicate SEM [Color figure can be viewed at wileyonlinelibrary.com]

$970 \mathrm{~nm}$ wavelength with a $\mathrm{XY}$ resolution of $0.6 \mu \mathrm{m}, \mathrm{Z}$ step of $3 \mu \mathrm{m}$. Images were analyzed and processed with Image $(\mathrm{NIH})$ software.

\subsection{0 | Unilateral nasal occlusion}

For olfactory deprivation, mice were subjected to unilateral nasal occlusion (UNO) as previously described (Cummings, Henning, \& Brunjes, 1997). UNO was performed on the right OB. Briefly, mice were anesthetized with ketamine/xylazine. Then, $2 \%$ lidocaine was applied locally onto the nostril. A polyethylene tubing (BD Intramedic, PE50) that was previously sealed with superglue was inserted in the nostril using Vaseline to facilitate the insertion. If necessary, an additional drop of superglue was applied on the outside to seal the nostril completely. UNO was verified weekly. Only animals with successful UNO were analyzed. UNO was maintained for 4 weeks.

\subsection{1 | Statistical analysis}

Statistical analyses were performed using R software (RRID:SCR_001905) and R Commander Package (https://CRAN.R-project.org/package=Rcmdr). Data are presented as mean \pm SEM. Two-tailed Mann Whitney $U$ test was performed for Figure 6 and Figure 7. Probability assignment: $p>0.05$ (not significant, ns), $0.01<p<0.05(*), 0.001<p<0.01(* *)$, and $p<0.001$ (***).

\section{3 | RESULTS}

\subsection{ND6 is specific to the glutamatergic lineage in the $\mathrm{OB}$}

To identify molecular markers and determinants of $O B$ neurons, we expressed GFP in the dorsal and lateral stem cell pools surrounding the lateral ventricles using targeted in vivo electroporation (Boutin et al., 2008). Subsequent microdissection and fluorescent-activated cell sorting of labeled cohorts at different time points postelectroporation was followed by microarray analysis. This approach led to a high-resolution picture of gene expression in the two lineages (Tiveron et al., 2017). Investigation of differentially expressed genes between both lineages showed that the bHLH transcription factor NeuroD6 (ND6) was strongly induced in the dorsal lineage between 1 and 4 days post-electroporation (dpe) but absent from lateral isolates (Figure 1a). We validated this expression for postnatal stages by quantitative real-time PCR (qRT-PCR) of micro-dissected dorsal or 


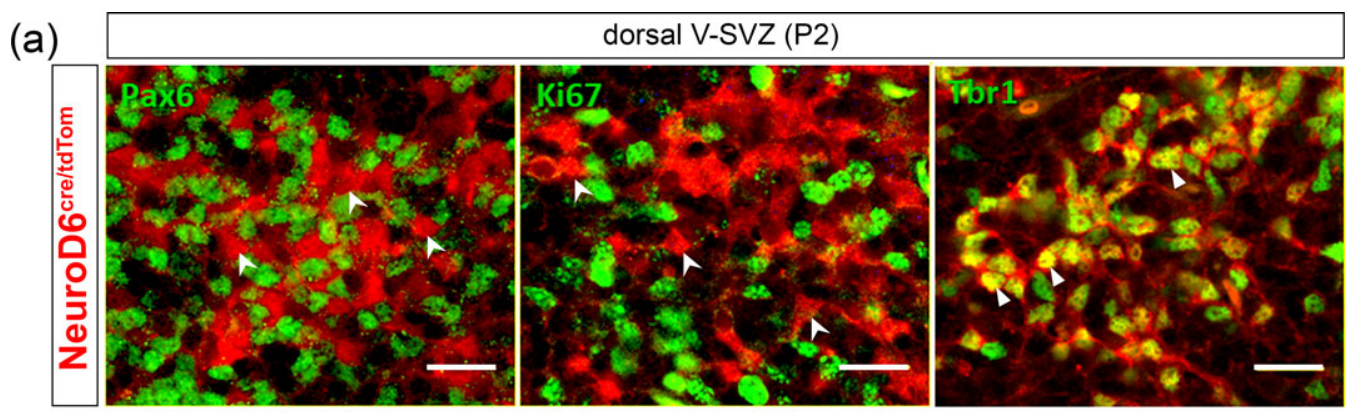

(b)

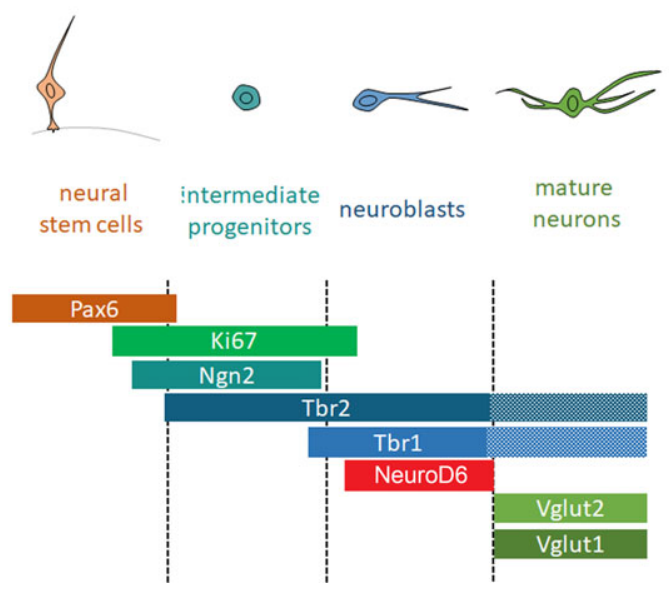

FIGURE 3 NeuroD6 is expressed in post-mitotic neurons. (a) Co-localization analysis of tdTom + cells with specific markers in dorsal V-SVZ of neonatal NeuroD6 ${ }^{\text {cre/tdTom }}$ mice. No co-localization with tdTom cells and stem cell marker Pax6 or proliferative marker Ki67 (gashed arrowheads). Full co-localization with tdtTom cells and post-mitotic marker Tbr1 (flat arrowheads). (b) Schematic representation of transcriptional cascade of glutamatergic neuron generation in the OB. Note that adult-born glutamatergic JGCs do not express Tbr2 (unknown for Tbr1) in mature stage. Scale bars: (a) $20 \mu \mathrm{m}$ [Color figure can be viewed at wileyonlinelibrary.com]

lateral V-SVZ as well as OB tissue. ND6 expression was undetectable when adult tissues were used (Figure $1 \mathrm{~b}$ ).

We aimed at characterizing the expression of ND6 in more spatial and temporal detail. In the absence of reliable antibodies against the transcription factor, we used constitutive ND6 ${ }^{\mathrm{Cre}}$ (Goebbels et al., 2006) and inducible ND6 ${ }^{\text {CreERT2 }}$ mice ((Agarwal et al., 2012); Schemes in Figure 1c) bred to a Cre-inducible Rosa26 $6^{\text {tdTomato(tdTom) }}$ reporter line (Madisen et al., 2010). In a constitutive ND6 ${ }^{\text {Cre }}$ background at P1, strong tdTom expression was observed in callosal axons, in agreement with the early expression of the TF in cortico-cortical projection neurons (Figure 1d; (Goebbels et al., 2006)). Moreover, axon bundles in the striatum (Figure 1d, arrow heads), likely representing corticostriatal connections, as well as individual cells in the septal region were tdTom positive. ND6 was generally absent from V-SVZ surrounding the lateral ventricles, except for a sparse cell population located in the dorsal aspect (Figure $1 d^{\prime}$ ). In the OB of ND6 ${ }^{\text {Cre/tdTom }}$ mice at P21 (Figure 1e), mitral and tufted cells were tdTom + , in agreement with previous observations (Goebbels et al., 2006). In addition, JGCs in the glomerular layer (GL) were labeled with tdTom. Quantification of these neurons demonstrated that ND6-derived cells represented $17.95 \pm 2.34 \%$ among all cells ( $n=3,885$ cells) within the GL.

Then, we aimed at identifying the neurotransmitter phenotype of

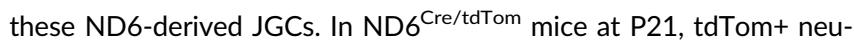
rons neither co-expressed GFP in a GAD67 ${ }^{\mathrm{GFP}}$ transgenic background nor were immunopositive for GAD65 staining (Figure 2a,b) indicating that they are not GABAergic. There was also no overlap with markers for specific GABAergic subtypes like CaIR or CalB (Figure 2c,d). Moreover, ND6 lineage cells also never expressed TH (Figure 2e), indicating that they are not dopaminergic.

Next, we used markers for glutamatergic neurons. In a constitu-

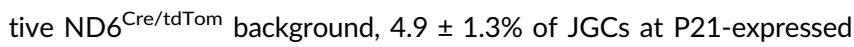
Tbr1 ( $n=3,225$ cells) and $71.3 \pm 6.9 \%$ were Tbr2 positive $(n=3$, 386 cells, Figure $2 \mathrm{f}, \mathrm{g}, \mathrm{j})$. Moreover, the vesicular glutamate transporters Vglut1 and Vglut2 were expressed in $66.7 \pm 2.9 \%$ and $42 \pm 6.5 \%$ of tdTom + JGCs, respectively ( $n=3,108$, and 146 cells, Figure $2 \mathrm{~h}-\mathrm{j}$ ). When exclusively perinatally born tdTom+ neurons were

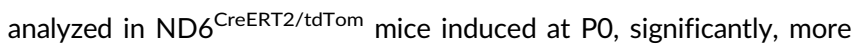
neurons were Tbr1 and Tbr2 positive (Tbr1 $26.8 \pm 8.2 \%, n=3$, 194 cells; Tbr2 $98.8 \pm 1.2 \%, n=3,195$ cells), and the ratio of Vglut1 to Vglut2 positive cells was inverted (Vglut1 $37.3 \pm 1.8 \%$ and Vglut2 $63 \pm 2.9 \%, n=3,241$, and 123 cells, respectively) (Figure 2j). Thus, ND6 ${ }^{\text {Cre }}$ labels embryonically and perinatally born glutamatergic neurons of the olfactory bulb.

To specifically analyze the generation and properties of perinatally born glutamatergic $O B$ neurons, we used inducible

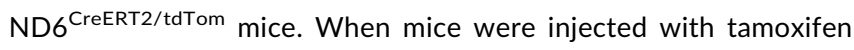
at $\mathrm{P} 1$ and analyzed 1 day post induction (dpi), most of the corpus callosum, striatum, and septum were devoid of tdTom staining (Figure 1f), thus facilitating the identification of individual tdTom+ cells. However, a small-cell population located in the dorsal V-SVZ strongly expressed tdTom (Figure $1 f^{\prime}$ ), comparable to the label in 
(a)

(b)
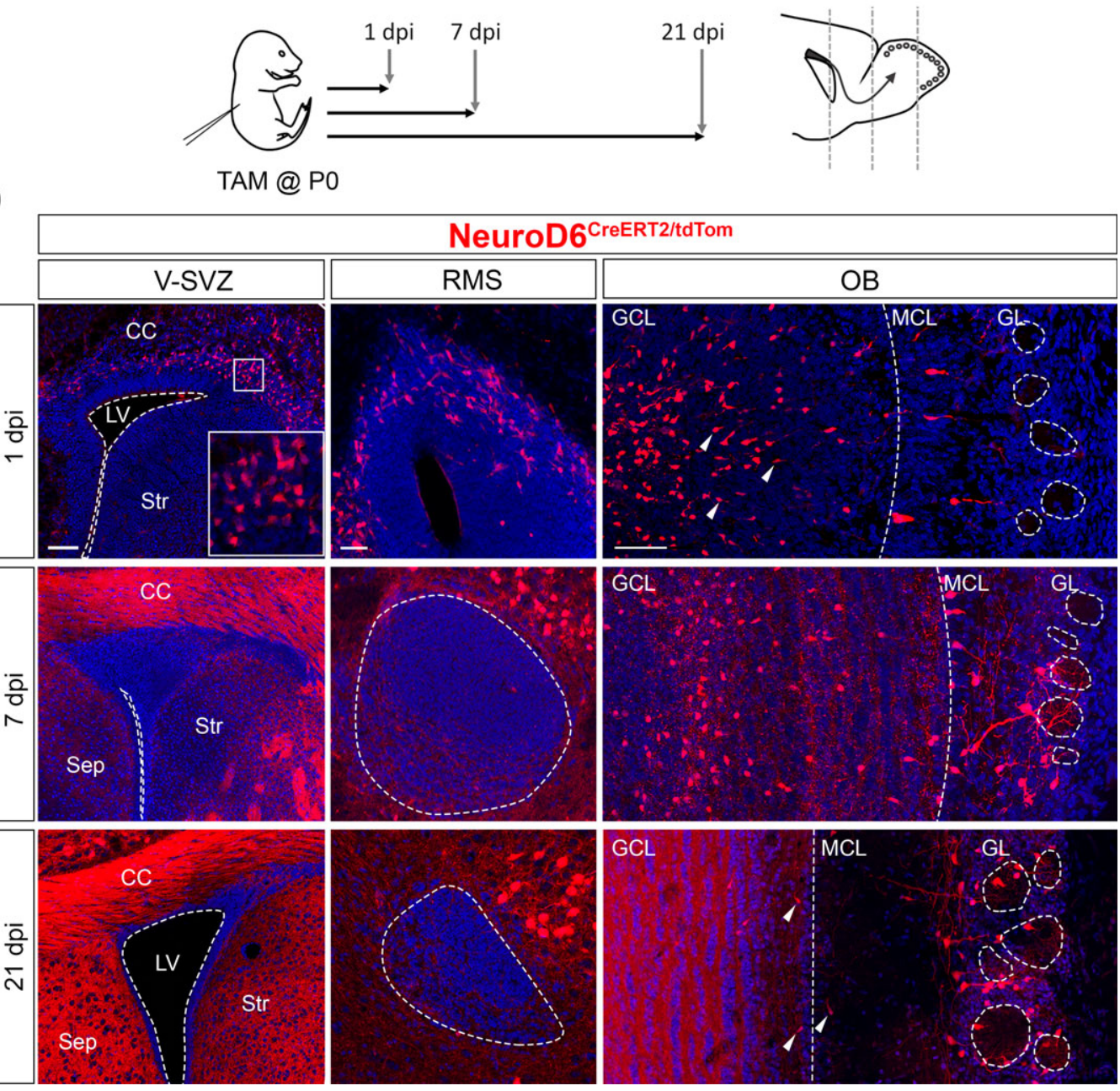

FIGURE 4 TAM induction in neonatal ND6 ${ }^{\text {CreERT2 }}$ mice recombines neuronal progenitors along the V-SVZ-RMS-OB axis. (a) Schematic representation of TAM-induced pulse-chase experiments. Pups were induced at PO and brains were harvested 1,7 , and 21 days post induction (dpi), respectively. Dotted lines on sagittal representation indicate the level of slice analysis from V-SVZ to OB. (b) The presence of recombined red cells along the V-SVZ-RMS-OB axis at different time points after TAM induction. At $1 \mathrm{dpi}$, neuronal progenitors are found in the dorsal $\mathrm{V}$ SVZ, in the RMS and in the deeper OB layers. Most cells display a migratory morphology (arrow head). At $7 \mathrm{dpi}$, recombined cells are found mainly within the OB. Cells with more complex morphology start to appear in the GL. At $21 \mathrm{dpi}$, cells are found almost exclusively within the GL and have acquired a mature neuronal morphology. Some cells are found close to the mitral cell layer (MCL, arrow head). RMS, rostral migratory stream. Scale bars: left to right: $100 \mu \mathrm{m}, 40 \mu \mathrm{m}, 100 \mu \mathrm{m}$ [Color figure can be viewed at wileyonlinelibrary.com]

constitutive $\mathrm{ND}^{\mathrm{Cre}}$ mice (Figure $\left.1 \mathrm{~d}, \mathrm{~d}^{\prime}\right)$. Immunostaining experiments revealed that all ND6-expressing V-SVZ cells co-expressed Tbr1 confirming that ND6 is specifically expressed in glutamatergic precursors (Figure 3a, flat arrowheads). However, ND6-tdTom+ V-SVZ cells never expressed the early marker Pax6 and were very rarely positive for $\mathrm{Ki} 67+(0.5 \pm 0.4 \%)$, showing that ND6 is confined to post-mitotic precursors (Figure $3 b$ ).

In the $\mathrm{OB}$, at $21 \mathrm{dpi}$, most mitral and tufted cells were tdTomnegative after induction (Figure 1g), indicating that ND6 is no longer expressed in these cells after birth. This result is also in agreement with the embryonic origin of $\mathrm{OB}$ projection neurons. In contrast, JGCs within the glomerular layer were tdTom+, like in constitutive

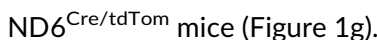

Next, we investigated the dynamics and potential lineage relation of the postnatal ND6 expressing populations in the dorsal V-SVZ, the RMS, and the OB. To this aim, we induced ND6 ${ }^{\text {CreERT2/tdTom }}$ mice with tamoxifen at P0 and analyzed the tdTom+ cell pool at 1, 7, and $21 \mathrm{dpi}$ at different rostro-caudal levels within the forebrain (Figure 4a).

Induction at PO and analysis at $1 \mathrm{dpi}$ led to the detection of labeled cells in the dorsal V-SVZ (Figure 4b, see also Figure 1f), in dorsal aspects of the developing RMS as well as in the center of the OB (Figure 4b). Immunostaining for the proliferation marker Ki67 and the post-mitotic neuron marker Tbr1 indicated that ND6 tdTom+ cells located in the RMS and the core of the $\mathrm{OB}$ are post-mitotic precursors of glutamatergic neurons (Supporting Information Figure S1, flat arrowheads). Moreover, at this time point, individual radial oriented cells in the GCL showed tdTom expression (Figure 4b, arrowhead). These cells had generally the spindle-like morphology of migratory neurons.

At $7 \mathrm{dpi}$, the thinning dorsal V-SVZ was devoid of tdTom positive cells, and fluorescent cells in the mid-RMS were observed only 
(a)

tdTom+ Group-1

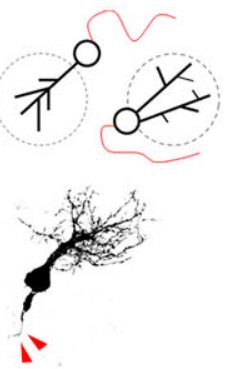

tdTom+ Group-2

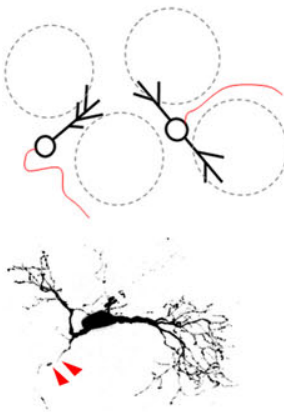

tdTom ${ }^{+}$Group-3

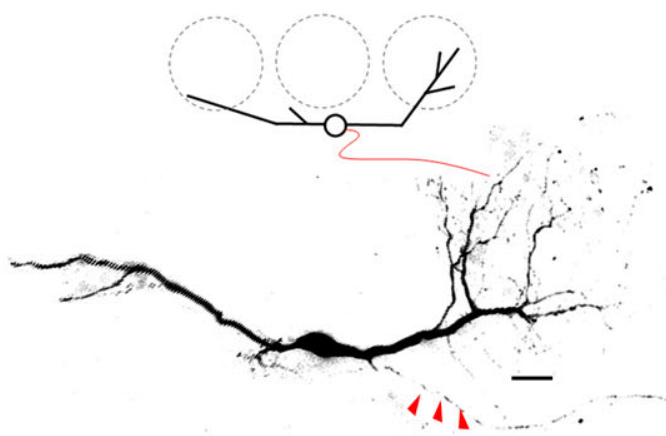

(b)

(d)

(c)

\section{Induction scheme}

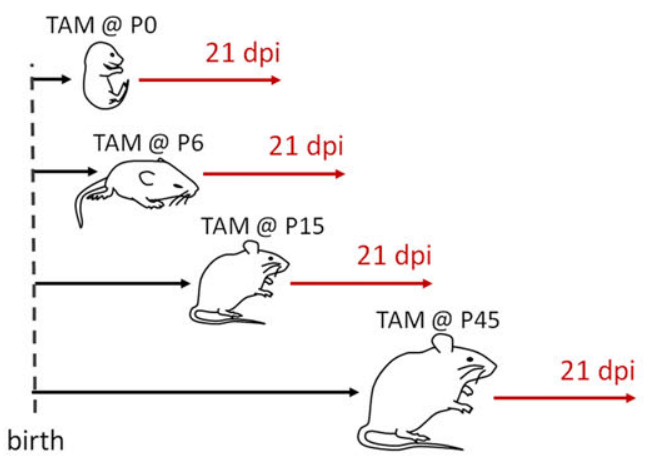

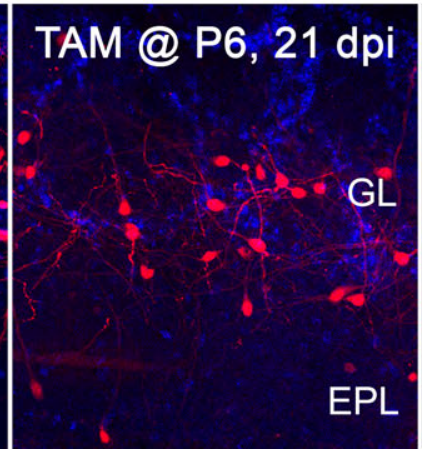

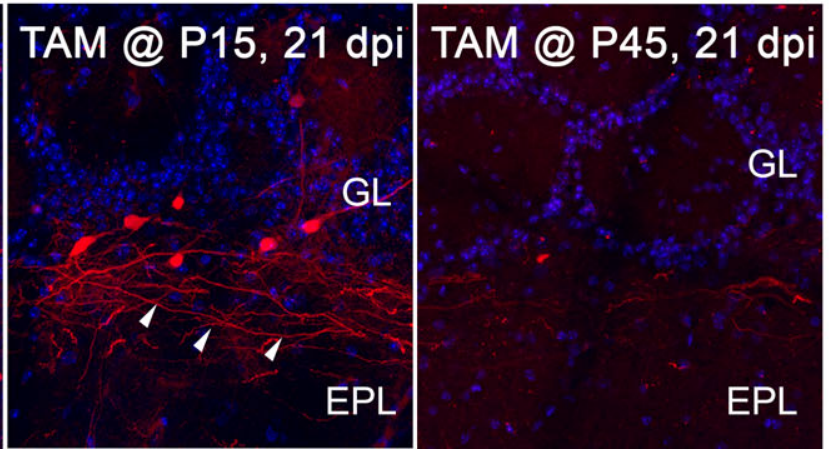

FIGURE 5 Glutamatergic juxtaglomerular cells (JGCs) are morphologically heterogeneous and can be classified into three main groups.

(a) Schematic representation (above) and real examples (below) of three main neuron groups based on their dendritic branching pattern into one glomerulus (Group-1), more than one glomerulus (Group-2) or the presence of an additional secondary dendrite extending into the EPL (Group-3). All categories display a fine protrusion, resembling an axon (indicated in red on scheme and with red arrows on real cells). (b) Schematic

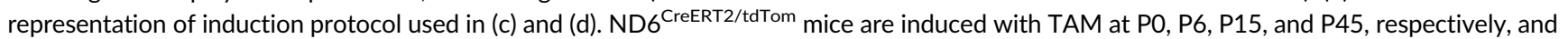
brains collected $21 \mathrm{dpi}$. (c) Quantification of categories of recombined glutamatergic JGCs when induction is performed at different time points. Note that a fourth group, short axon cells (SAC), is also detected. However, given that this cell type is seldom observed and that dendritic morphology does not correspond to the classical description, we did not further quantify these cells. (d) Representative confocal images of animals induced at different time points showing enlarged view of the GL and EPL. The amount of recombined red cells declines progressively as induction is performed in older animals. Scale bars: (a) $20 \mu \mathrm{m}$, (d) $100 \mu \mathrm{m}$ [Color figure can be viewed at wileyonlinelibrary.com]

sporadically (Figure 4b). However, many cells were still found in the rostral RMS, within the $\mathrm{OB}$, and in the GCL. At this time point, cells with neuronal morphology appeared in the peripheral layers of the $\mathrm{OB}$, the mitral cell layer, the EPL, and the GL.

When PO-induced brains were analyzed at $21 \mathrm{dpi}$, the V-SVZ, the RMS, and the GCL were devoid of tdTom positive cells, whereas many cells with mature neuronal morphology were now observed in the peripheral layers of the OB (Figure $4 b$ ). We interpret that ND6 is expressed in lineage-related progenitors and migratory neuroblasts in the V-SVZ and RMS that eventually settle in the GL. However, other scenarios, like persistent local generation of glutamatergic neurons within the $\mathrm{OB}$, appear possible.

To investigate this issue of generation of glutamatergic JGCs by V-SVZ-derived progenitors as opposed to intrabulbar progenitors, we performed localized intraventricular injection of hydroxytamoxifen (4OH-TAM) in PO ND6 ${ }^{\text {CreERT2/tdTom mice. At a concentration of }}$ 
(a)

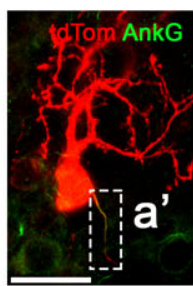

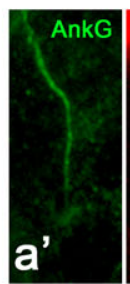

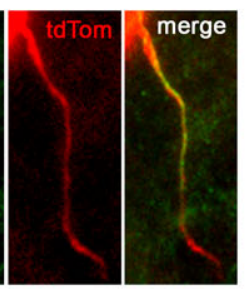

(b)

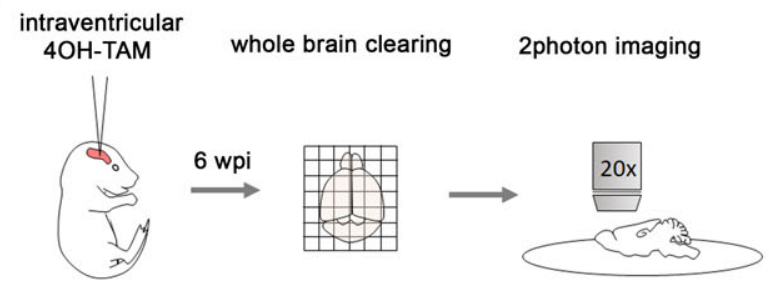

(c) type-1 axonal projection (superficial)
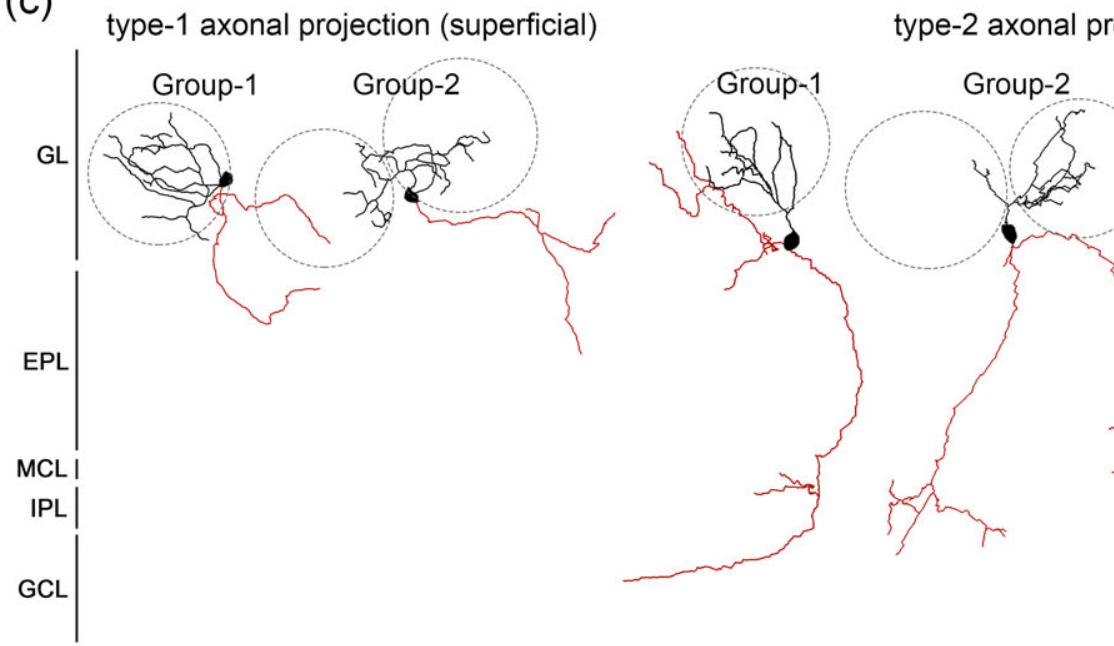

type-2 axonal projection (deep)

(d)

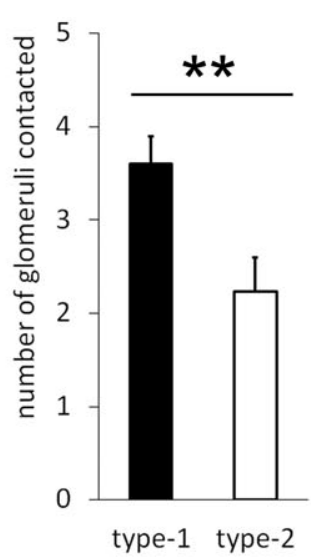

(e)

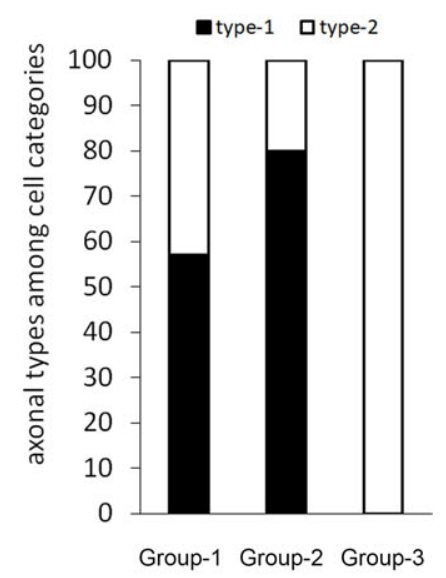

(f)

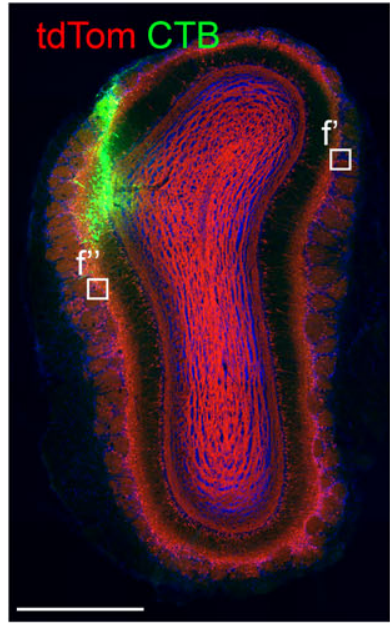

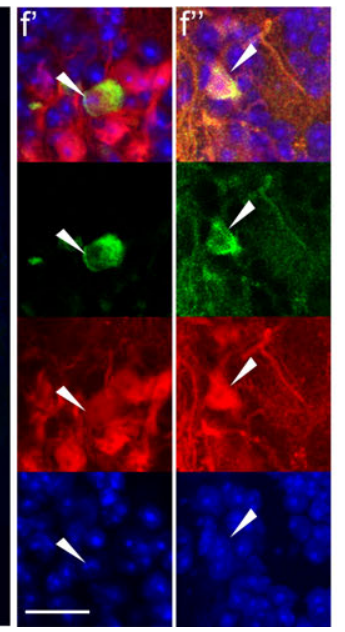

FIGURE 6 Glutamatergic JGCs project axons across all layers of the olfactory bulb. (a) tdTom+ cells display thin protrusions which are immunoreactive for axonal initial segment marker Ankyrin G (AnkG). Detail of co-localization shown in (a'). (b) Schematic representation of experimental setup to induce sparse labeling and follow axonal projections. Intraventricular hydroxytamoxifen (4OH-TAM) injection in neonatal

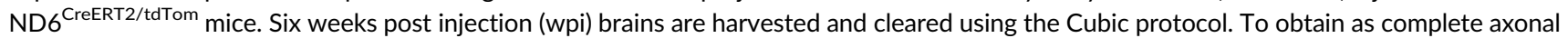
reconstructions as possible, entire transparent brains were imaged using two-photon microscopy. (c) Partial axonal reconstructions of the different postnatal generated glutamatergic JGCs. Axonal projections (red) either remain within the glomerular layer (type-1 superficial axons) or dive into the GCL (type-2 deep axons) where they cannot be traced anymore due to high density of projections. Deep axons are frequently observed to specifically branch within the inner plexiform layer (IPL). (d) Type-1 axons contact significantly more glomeruli than type-2 axons $\left(p=0.0098, * * p<0.01\right.$, two-tailed Mann Whitney $U$ test, $\left.n_{\text {(superficial/deep }}\right)=25 / 17$ cells, three animals). (e) Relative occurrence of axonal types on glutamatergic JGC categories. Type-1 and type-2 axons appear on external tufted cells (Group-1 and 2). All traced superficial tufted cells (Group3) displayed type-2 axons ( $\left.n_{(G r o u p-1 / 2 / 3}\right)=28 / 10 / 4$ cells, three animals). ( $f$ ) Retrograde labeling of tdTom+ cells (medial) after localized CTB injection (lateral) suggests that type- 2 axons connect to the opposite side within the same OB. ( $f^{\prime}$ ) Double-labeled CTB+tdTom+ cells are systematically found on OB side opposite to the injection site (59 cells, three animals) but also adjacent ( $\left.f^{\prime \prime}\right)$ to the injection site. Scale bars: (a) $20 \mu \mathrm{m}$; (f), $1 \mathrm{~mm}$; (f') $20 \mu \mathrm{m}$. Error bars indicate SEM [Color figure can be viewed at wileyonlinelibrary.com]

$500 \mu \mathrm{M}, 4 \mathrm{OH}-\mathrm{TAM}$ injection led to recombination of progenitors exclusively in the V-SVZ (Supporting Information Figure S2a) but not to the appearance of tdTom+ cells in the center of the $\mathrm{OB}$, at $1 \mathrm{dpi}$
(Supporting Information Figure S2b). At $21 \mathrm{dpi}$, the V-SVZ was devoid of tdTom + cells (Supporting Information Figure S2c), whereas we robustly found recombined tdTom+ JGCs in the OB (Supporting 
(a)

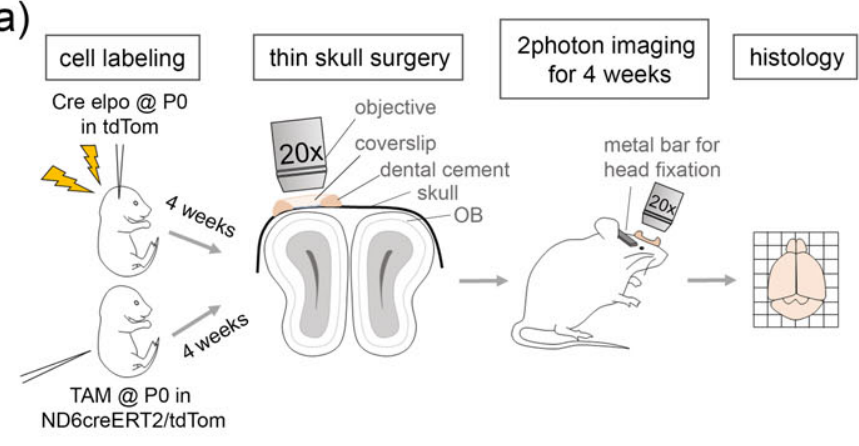

(b)
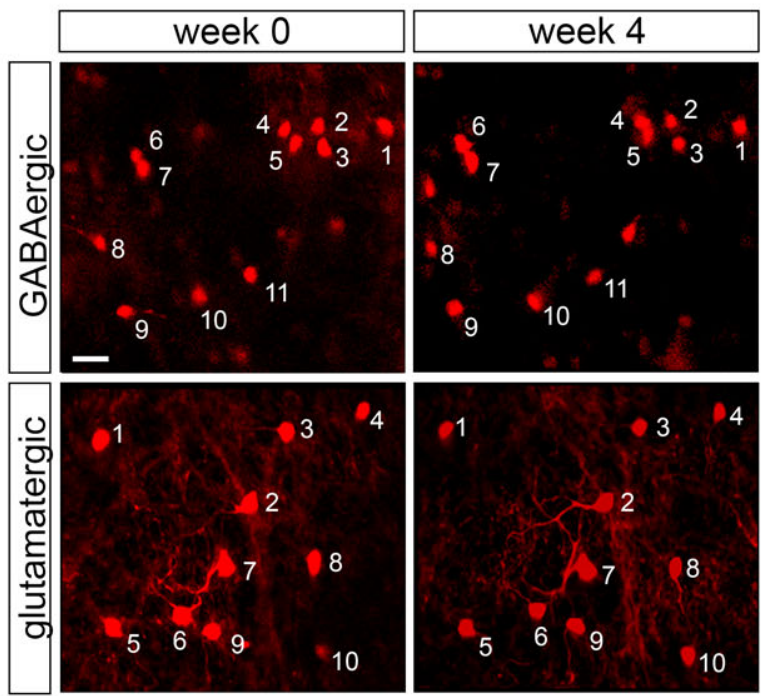

(c)

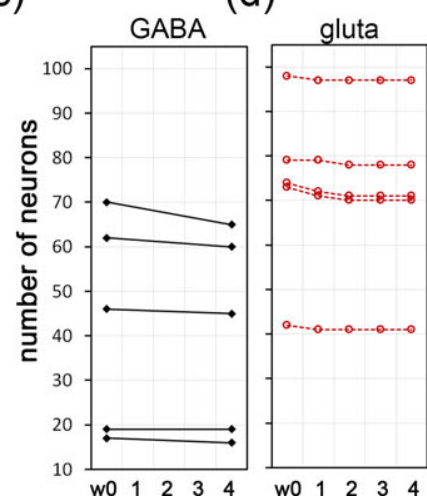

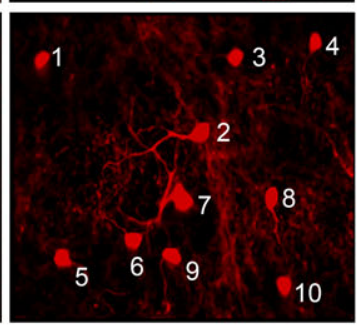

(e)

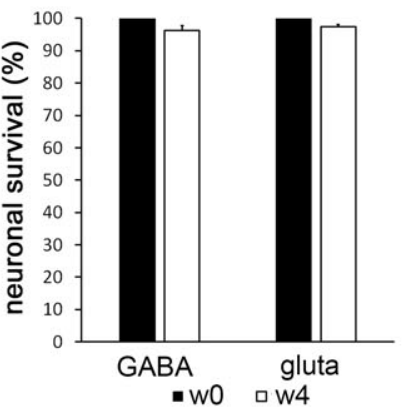

(f)

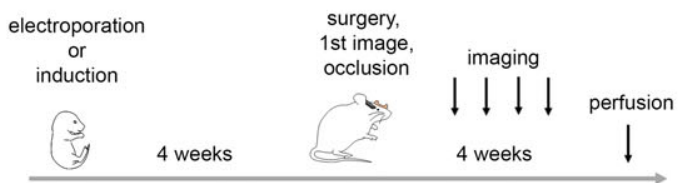

(g)

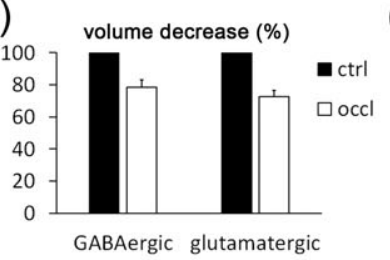

(h)

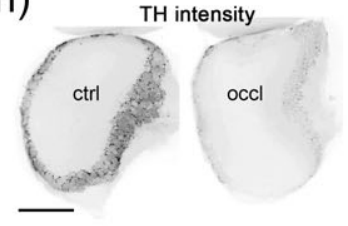

(i)
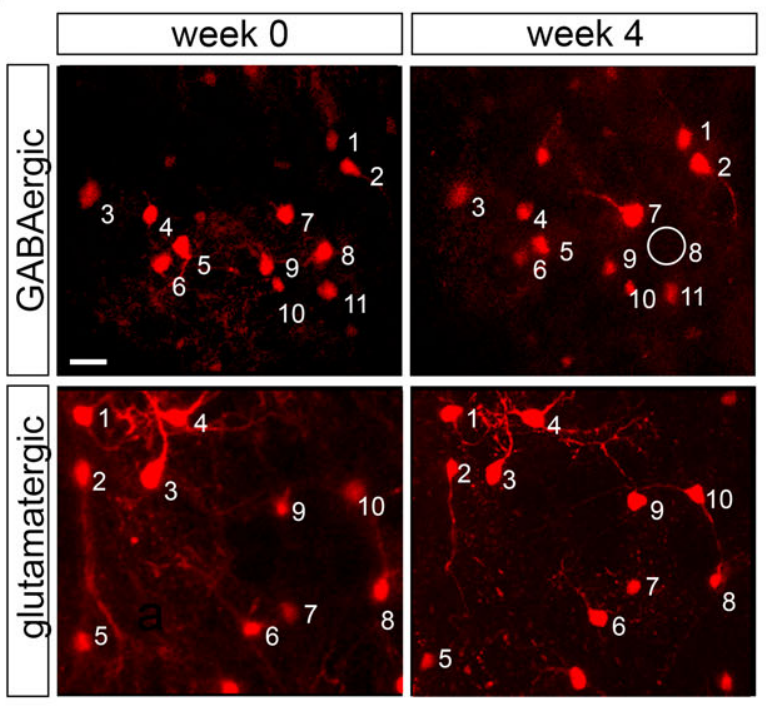

(j)

(k)

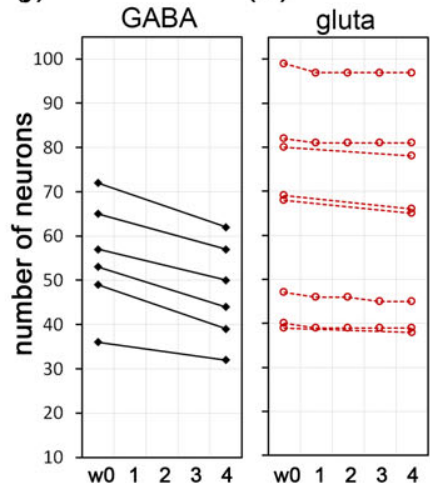

(I)

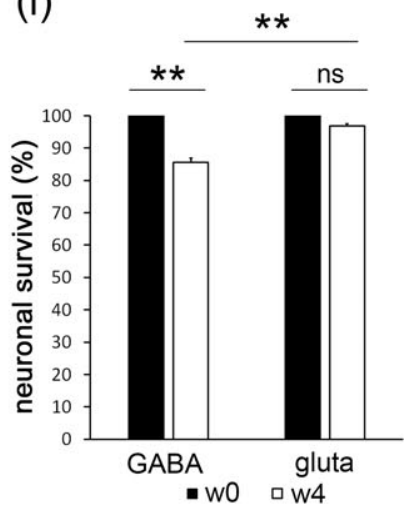

FIGURE 7 Glutamatergic JGCs are resistant to sensory deprivation. (a) Schematic representation of experimental procedure carried out to study in vivo survival of glutamatergic and GABAergic cells. To mark GABAergic JGCs, Cre recombinase plasmid was electroporated (elpo) into PO tdTom reporter mice. To mark glutamatergic JGCs ND6 ${ }^{\text {creERT2/tdTom }}$ was induced at PO. Four weeks after elpo or induction, thin-skull surgery was performed. Mice were imaged for 4 weeks using a two-photon microscope. Their brains were subsequently fixed and processed for histological analysis. (b) Image of the same region for GABAergic cells (above) and glutamatergic JGCs (below), short after tdTom recombination (week0) and 4 weeks later (week4). Each cell is numbered and identified based on their relative position to other cells and distinctive dendritic branching pattern (whenever possible). (c) Number of GABAergic JGCs followed in individual animals (black lines) in the course of 4 weeks. (d) Number of glutamatergic neurons followed in individual animals (red lines) in the course of 4 weeks. (e) Percentage of GABA- and glutamatergic JGCs that survived under control condition in the course of 4 weeks as compared to week 0 . (f) Schematic representation of experimental setup conducted for sensory deprivation using unilateral naris occlusion. The scheme recapitulates essentially the protocol depicted in (a) with the difference that in a group of animals, unilateral naris occlusion was additionally performed at the moment of the surgery. (g) Loss of approximately $30 \%$ of OB volume in occluded (occl) OB as compared to control OB (ctrl). (h) Strong decrease in TH immunoreactivity further confirms successful occlusion. (i) Image before (week 0) and 4 weeks after (week 4) occlusion of the same region for GABAergic cells (above) and glutamatergic JGCs (below). Note that under occlusion, a fraction of GABAergic cells disappears (circle). (j) Number of GABAergic JGCs followed in individual animals (black lines) during 4 weeks of occlusion. (k) Number of glutamatergic neurons followed in individual animals (red lines) during 4 weeks of occlusion. (I) Percentage of GABA- and glutamatergic JGCs that survived under occlusion in the course of 4 weeks as compared to week 0 . Although a significant decrease in GABAergic cell number is observed $\left(p=0.00804\right.$ for $\mathrm{GABA}_{\text {ctrl }}$ vs $\mathrm{GABA}_{\text {occl }}, p=0.0018$ for $\mathrm{GABA}_{\text {occl }}$ vs gluta occl , ** $p<0.01$, two-tailed Mann Whitney $U$ test, $n_{\mathrm{GABA}(\mathrm{ctr} / \mathrm{occl})}=214 / 279$ cells, $5 / 6$ animals, respectively), glutamatergic cell number remains stable $\left(n_{\text {Gluta(ctrl/occl) }}=365 / 403\right.$ cells, $5 / 9$ animals, respectively). Scale bars: (b) $20 \mu \mathrm{m}$; (h) $1 \mathrm{~mm}$; (i) $20 \mu \mathrm{m}$. Error bars indicate SEM [Color figure can be viewed at wileyonlinelibrary.com] 
Information Figure S2d). This indicates that even if the majority of glutamatergic JGCs born around PO likely originate from remaining intrabulbar progenitors, a subset is generated from V-SVZ progenitors.

Altogether the combined use of gene expression analyses and Cre-loxP-based lineage tracing leads to the conclusion that NeuroD6 is specifically but transitorily expressed in a subpopulation of perinatally generated neuronal precursors that migrate from the dorsal aspect of the V-SVZ to peripheral layers of the OB to differentiate into glutamatergic neurons.

\section{2 | Glutamatergic OB neurons are generated early after birth}

Morphological characterization of labeled JGCs in ND6 ${ }^{\text {CreERT2/tdTom }}$ mice 21 days after induction at PO identified different groups of tdTom+ cells. The first group extends complex-branched dendrite trees into a single glomerulus and is interpreted as external tufted cells (ETCS). The second group resides within the $\mathrm{GL}$ and sends branched dendrites into two or more glomeruli and is referred to as external bi-tufted cells (biETCs). Furthermore, we identified a third group of tdTom + cells that, in addition to their complex primary tuft, extend a sparsely branched secondary dendrite into the EPL (Figure 5a). These cells are located at the border between the GL and the EPL and resemble superficial tufted cells. We also observed few cells in juxtaglomerular positions that extend sparsely branched dendrites into the interglomerular space, resembling previously described short axon cells (SACs, Figure 5c), as well as some small-soma sized cells that reside in the MCL (Brill et al., 2009; Hayar, Karnup, Shipley, \& Ennis, 2004b; Liu \& Shipley, 2008; Winpenny et al., 2011).

To gain insight into the temporal generation dynamics of these postnatal generated types, we induced Cre expression from the NeuroD6 locus by tamoxifen injection at different time points and analyzed the morphology of the induced cohorts 21 days later, after their arrival and integration in the $\mathrm{OB}$ (Figure $5 \mathrm{~b}$ ). When induced at PO, we typically observed about seven fluorescent neurons per glomerulus. Over all analyzed glomeruli, this population consisted of about $61 \%$ ETCs, 9\% biETCs, $26 \%$ superficial TCs, and 4\% SACs (Figure $5 c, d$ ). After induction at P6, generation of ETCs dropped relative to the other cell types leading to a relatively even distribution of the cell types. After induction at $\mathrm{P} 15$, production of all three subtypes decreased considerably (Figure $5 \mathrm{c}, \mathrm{d}$ ) and superficial TC processes in the EPL became most prominent (Figure $5 d$, arrow head). After induction at P45, only very few cells (nine cells in four animals) with lateral dendrites that extend into the EPL were identified (Figure $5 \mathrm{~d}$ ), showing that the adult generation of glutamatergic OB cells represents a rare exception.

\section{3 | Glutamatergic JGCs project axons across the $\mathrm{OB}$}

Perinatally generated ND6-derived JGCs displayed along with their dendritic trees also thin axon-like processes (arrows in Figure 5a). Using the AIS marker Ankyrin G, we doubtlessly confirmed axonal identity (Figure 6a). Indeed, all ND6-derived neurons had axons. Next, we aimed at describing the axonal projection pattern of the different perinatally generated glutamatergic cell populations in detail. We performed intraventricular hydroxytamoxifen (4OH-TAM) injection in P0

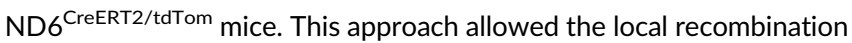
of glutamatergic progenitors lining the lateral ventricle in the absence of contaminating centrifugal cortical projections into the OB. Animals were perfused 6 wpi when neurons had fully integrated into their target layer within the OB. As complex axons can hardly be observed in standard tissue sections, we performed tissue clearing of entire brains using the CUBIC protocol (Susaki et al., 2015) and imaged transparent brains with two-photon microscopy (for experimental setup, see Figure $6 \mathrm{~b}$ ). Tracing of axons of individual external tufted and bi-tufted neurons revealed the existence of two distinguishable subgroups based on their projection pattern (Figure 6c). First, we observed type1 axonal projections of ETCs and biETCs that extend only within superficial layers (GL and EPL). These represent $57 \%$ and $80 \%$ of the total population, respectively (Figure $6 e, n=3,38$ cells traced). Second, type-2 projecting ETCs and biETCs, in addition to superficial axons, send projections into the deep layers including the GCL $(43 \%$ and 20\%, respectively; Figure 6c,e). Type-1 axonal projections cover a significantly wider array of glomeruli than type-2 axons (Figure 6d).

Previous retrograde labeling has demonstrated that these subtypes of ETC did not project outside the bulb and were thus considered as interneurons (Schoenfeld \& Macrides, 1984).

Finally, superficial TCs always had deep (type-2) projections that branched extensively in the IPL and GCL ( $n=3,4$ cells, Figure $6 \mathrm{c}, \mathrm{e}$ ) and are likely true projection neurons (Schoenfeld \& Macrides, 1984; Schoenfeld, Marchand, \& Macrides, 1985).

Then, we aimed at investigating the intrabulbar projection site of the axonal processes of glutamatergic JGCs. However, despite the use of clearing protocols and two-photon microscopy, tracing of individual tdTom positive axons was limited due to high density of axons within the GCL. Therefore, we performed a retrograde-labeling approach. The retrograde tracer cholera toxin subunit B (CTB) was locally injected into the lateral aspect of the OB of 6 weeks old ND6 ${ }^{\text {Cre/tdTom }}$ animals ( $n=3$, Figure $6 f$ ). One week later, CTB/tdTom double positive cells were systematically found in proximity of the injection side, indicating the presence of type-1 JGCs with superficially branching axons (Figure $6 f^{\prime \prime}$ ). In addition, double positive cells (approximately 60 cells per animal within a Z-depth of $150 \mu \mathrm{m}$ ) were identified within the GL opposite to the injection site, that is, on the medial aspect of the ipsilateral OB (Figure $6 f^{\prime}$ ). We conclude that glutamatergic JGCs project complex axonal projections, often over considerable distances, throughout the OB. Therefore, postnatally ND6-derived JGCs represent a mixed population of interneurons positioned within the $G L$ and projection neurons located at the border between the $G L$ and the EPL,

\section{4 | Glutamatergic JGCs survive sensory deprivation}

Several studies demonstrated sensitivity of GABAergic and dopaminergic JGCs to environmental stimuli (Baker, 1990; Kato et al., 2012; Mandairon, Jourdan, \& Didier, 2003; Sawada et al., 2011). Indeed, sensory deprivation by naris closure leads to extensive neuronal death among neonatal and adult born inhibitory interneurons of the $\mathrm{OB} \mathrm{GCL}$ 
and GL. Excitatory interneurons have not been studied in this context.

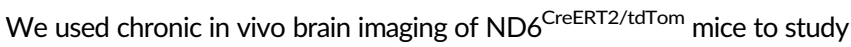
the long-term survival of glutamatergic JGCs under normal conditions and under sensory deprivation. To induce a control cohort of mostly GABAergic JGCs, for which deprivation-sensitivity has been demonstrated, we performed postnatal (PO) electroporation of a Cre expressing DNA plasmid into the dorsal ventricular wall of Rosa $26^{\text {tdTomato }}$ mice. This approach labeled a mixed population of GABAergic and GABA/dopaminergic JGCs but only scarcely glutamatergic JGCs (Tiveron et al., 2017). In parallel, we induced postnatally born cohorts of tdTom expressing glutamatergic JGCs by TAM injection in ND6 ${ }^{\text {CreERT2/tdTom mice at PO. Four }}$ weeks after induction or electroporation, thin-skull surgery (Shih et al., 2012; Yang et al., 2010) was performed above the relevant OB. A field of view containing large amounts of fluorescent neurons was chosen, and a volume of $600 \times 600 \mu \mathrm{m}$ in $\mathrm{XY}$ and $200 \mu \mathrm{m}$ in $Z$ was imaged at high resolution with a two-photon microscope (Figure 7a). This approach provided high-resolution images of the GL but not the underlying EPL, excluding superficial TCs from the analysis. Regular imaging over 4 weeks allowed the in vivo observation of neurons under both labelling schemes (Figure 7b). Neurons were individually identified, numbered, and revisited several times over the observation period (Figure $7 b-d$ ). These data demonstrated that under normal conditions, cells labeled by both CRE induction protocols, postnatal dorsal electroporation, or injection of ND6 ${ }^{\text {CreERT2/tdTom }}$ mice were extremely stable over time and neuronal loss was quasi absent (Figure $7 b-e$ ).

Next, we performed the same labelling approaches but combined with unilateral naris occlusion to cause sensory deprivation (Figure $7 f$ for experimental setup). As previously reported (Sawada et al., 2011), the occluded $O B$ side significantly shrunk (Figure 7g) and lost a considerable amount of TH immunoreactivity as a consequence of successful naris occlusion (Figure 7h). GABA- and dopaminergic JGCs labeled by dorsal electroporation showed a significant cell loss of $13 \%$ (279 cells in six animals, Figure 7i,j,l). In contrast, among the 403 glutamatergic JGCs followed over the 4 weeks deprivation period, only 13 cells were not clearly identifiable in subsequent imaging sessions and therefore labeled as "dead." Thus survival of glutamatergic neurons was unaffected as compared to the control condition (Figure 7k,I).

Thus, although a considerable sub-fraction of perinatally born GABA- and dopaminergic JGCs are sensitive to sensory deprivation, perinatally born glutamatergic JGCs are resistant and survive even in the absence of environmental stimulation.

\section{4 | DISCUSSION}

In the present study, we show that the bHLH transcription factor NeuroD6 represents a novel and reliable marker for glutamatergic neurons in the OB. We therefore exploited ND6 $6^{\mathrm{Cre}}$ and ND6 ${ }^{\mathrm{CreERT} 2}$ mice as genetic tools to study this elusive cell population. We found that ND6 expression in the V-SVZ-RMS-OB system is transient and confined to immature progenitors. Induction of ND6 ${ }^{\text {CreERT2 }}$ labels therefore a well-defined and timed cohort. Next, we demonstrated that glutamatergic JGCs are not only heterogeneous in dendritic arborization but also in axonal projection patterns. Lastly, we showed that other than inhibitory JGCs, glutamatergic JGCs resist sensory deprivation.

\subsection{ND6 expression is transient and confined to glutamatergic $O B$ progenitors}

ND6 expression has been described for a multitude of brain areas including the neocortex, hippocampus, as well as some mid- and hindbrain structures (Goebbels et al., 2006). Interestingly, although studies that have investigated ND6 expression in detail agree upon the fact that promoter activity starts at the level of post-mitotic progenitors, expression maintenance varies greatly according to brain area and possibly protein function (Goebbels et al., 2006; Kay, Voinescu, Chu, \& Sanes, 2011; Wu et al., 2005). Using a battery of markers in the V-SVZ we found no co-localization of tdTom with the stem cell marker Pax6, rare co-localization with intermediate progenitor marker Ki67, and full overlap with post-mitotic marker Tbr1. This indicates that also in the postnatal V-SVZ, ND6 is expressed in post-mitotic progenitors (Brill et al., 2009). These results are further corroborated by our observations from lineage tracing experiments with

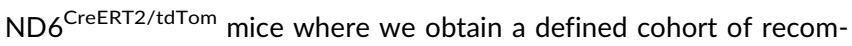
bined neurons after induction. Moreover, the lack of recombined cells within the VZ with radial glia-like or ependymal cell morphology argues against the possibility that ND6 is expressed in RGCs at their last round of division before becoming ependymal cells. Finally, the absence of fluorescent neurons within the GL at 1 dpi indicates that ND6 promoter is not active once neuroblasts reached their target layer. Altogether, these data show that ND6 is transiently expressed starting from post-mitotic progenitors and stopping before final neuronal integration.

\section{2 | Glutamatergic JGCs are morphologically heterogeneous and project axons across the $O B$}

The OB is dominated by inhibitory transmission, with the vast majority of neurons being GABAergic (Burton, 2017; Parrish-Aungst et al., 2007). However, locally connecting excitatory OB neurons like short axon cells (Aungst et al., 2003) and external tufted and bi-tufted cells (Hayar, Karnup, Ennis, et al., 2004; Hayar, Karnup, Shipley, et al., 2004) constitute an important part of the OB circuitry. With the emergence of lineage tracing in mouse mutants, two studies have shown that these JGCs are also produced postnatally (Winpenny et al., 2011) and to a lesser extent even in adult (Brill et al., 2009). In agreement, we show that external tufted, bi-tufted cells and to a lesser extend superficial tufted cells are indeed generated at perinatal stages. Moreover, we show that the majority of glutamatergic perinatally born JGCs are ETCs and that the overall neuron production drops drastically and rapidly after birth.

We provide evidence for the existence of two types of axonal projections in perinatally born JGCs; type-1 projections that remain within the glomerular layer and type- 2 projections that contact less glomeruli and dive deep into the core of the OB. Moreover, our retrograde tracing shows that some glutamatergic JGCs project even across the medio-lateral aspects of the $\mathrm{OB}$. This finding suggests that 
type-2 projections belong to the intrabulbar associated system (Liu \& Shipley, 1994) or mirror-symmetric isofunctional odor columns (Grobman et al., 2018). This intrabulbar circuit typically connects two glomeruli that express the same odorant receptor in a medio-lateral manner and is mediated by ETCs that project through the GCL and synapse with GCs situated just underneath its associated glomerulus on the other side of the OB (Liu \& Shipley, 1994). However, although we did not provide any experimental evidence for extrabulbar projection, it is likely that part of the type- 2 superficial TCs project outside the bulb, particularly in the pars externa of the anterior olfactory nucleus (Schoenfeld \& Macrides, 1984).

\section{3 | Perinatally born glutamatergic JGCs resist sensory deprivation}

How does environmental information impact glutamatergic JGCs? Sensory deprivation by unilateral naris occlusion is a powerful approach to investigate this question within the $O B$ brain circuitry. There is a well-established body of literature that shows how olfactory sensory deprivation causes cell death throughout all OB layers (Mandairon, Sacquet, Jourdan, \& Didier, 2006; Najbauer \& Leon, $1995)$ thereby emphasizing the importance of sensory input for survival of OB inhibitory interneurons (Petreanu \& Alvarez-Buylla, 2002; Rochefort, Gheusi, Vincent, \& Lledo, 2002; Yamaguchi \& Mori, 2005). We use a chronic in vivo imaging approach to directly monitor the survival of a to-date unexplored $O B$ neuron population, glutamatergic JGCs. In the first imaging session, we identify individual fluorescently labeled GABAergic and glutamatergic neurons and follow them over time in control situation and under occlusion. We demonstrate that although a significant fraction of GABAergic JGCs disappear, the vast majority of glutamatergic JGCs remain in place under unilateral naris occlusion. Many studies have demonstrated that a fraction of GABAergic cells undergo cell death under occlusion (Bastien-Dionne et al., 2010; Bovetti et al., 2009; Kato et al., 2012; Parrish-Aungst et al., 2011; Sawada et al., 2011). But why does this apparently general phenomenon not apply to glutamatergic JGCs? The most obvious difference between these two cell types clearly is reflected in their neurotransmitter phenotype and thus their function in the $\mathrm{OB}$ as either excitatory or inhibitory circuit elements. It is intriguing to view the $O B$ system as an intricate homeostatic balance between excitation and inhibition, finely tuned by the environmental challenges imposed to the system. In this scenario, the decreased environmental excitation has to be compensated by an overall decrease in bulbar inhibition, allowing the system to still confer information to higher order brain areas. Because adult neurogenesis provides the OB continuously with inhibitory interneurons, directed apoptosis could be a feasible mechanism to fine-tune inhibitory elements of the OB system. In the meantime, excitatory elements have to adjust their excitability too. However, given that adult neurogenesis does not supply the $O B$ with a significant amount of glutamatergic neurons at later ages, it seems unlikely that apoptosis could be a regulative mechanism for adjustment of excitation.

Further experiments will be needed to elucidate why cell death occurs selectively to some elements of the $O B$ circuit and not to others. However, our results represent a first step toward a more differentiated view of the OB network where both, local excitation and as well as inhibition are provided and adjusted by the interplay of an intricately complex network with its environment.

\section{ACKNOWLEDGMENTS}

We thank Sandra Goebbels and Markus Schwab for NeuroD6 ${ }^{\text {Cre }}$ and NeuroD6 $6^{\text {CreERT2 }}$ animals, and Yuchio Yanagawa for Gad67 $7^{\text {GFP }}$ mice. We are particularly grateful to the local PiCSL-FBI core facility (IBDM, AMU-Marseille) supported by the French National Research Agency through the "Investments for the Future" program (France-Biolmaging, ANR-10-INBS-04) as well as the IBDM animal facilities. This work was supported by fellowships from the Biotrail program and the Fondation pour la Recherche Medicale (FRM) to A. Angelova. H. Cremer was supported by Agence National de la Recherche (grant ANR-13-BSV4-0013), Fondation pour la Recherche Médicale (FRM) grants ING20150532361 and FDT20160435597, Fondation de France (FDF) grant FDF70959, and the Association France Parkinson.

\section{AUTHOR CONTRIBUTIONS}

$\mathrm{AA}, J \mathrm{CP}, \mathrm{HC}$, and NC developed the concept and designed the experiments. $A A$ acquired and analyzed the data with the help of JCP. CB designed, conducted, and analyzed the qRT-PCR experiment. AA made the figures. $\mathrm{AA}, \mathrm{HC}$, and $\mathrm{NC}$ wrote the manuscript.

\section{CONFLICT OF INTEREST}

The authors declare no sources of interest.

\section{ORCID}

Nathalie Coré (D) https://orcid.org/0000-0003-3865-4539

\section{REFERENCES}

Agarwal, A., Dibaj, P., Kassmann, C. M., Goebbels, S., Nave, K. A., \& Schwab, M. H. (2012). In vivo imaging and noninvasive ablation of pyramidal neurons in adult NEX-CreERT2 mice. Cerebral Cortex, 22, 1473-1486.

Antal, M., Eyre, M., Finklea, B., \& Nusser, Z. (2006). External tufted cells in the main olfactory bulb form two distinct subpopulations. The European Journal of Neuroscience, 24, 1124-1136.

Aungst, J. L., Heyward, P. M., Puche, A. C., Karnup, S. V., Hayar, A., Szabo, G., \& Shipley, M. T. (2003). Centre-surround inhibition among olfactory bulb glomeruli. Nature, 426, 623-629.

Baker, H. (1990). Unilateral, neonatal olfactory deprivation alters tyrosine hydroxylase expression but not aromatic amino acid decarboxylase or GABA immunoreactivity. Neuroscience, 36, 761-771.

Bastien-Dionne, P. O., David, L. S., Parent, A., \& Saghatelyan, A. (2010). Role of sensory activity on chemospecific populations of interneurons in the adult olfactory bulb. J Comp Neurol, 518, 1847-1861.

Boutin, C., Diestel, S., Desoeuvre, A., Tiveron, M. C., \& Cremer, H. (2008). Efficient in vivo electroporation of the postnatal rodent forebrain. PLoS One, 3, e1883.

Bovetti, S., Veyrac, A., Peretto, P., Fasolo, A., \& De Marchis, S. (2009). Olfactory enrichment influences adult neurogenesis modulating GAD67 and plasticity-related molecules expression in newborn cells of the olfactory bulb. PLoS One, 4, e6359. 
Brill, M. S., Ninkovic, J., Winpenny, E., Hodge, R. D., Ozen, I., Yang, R., ... Gotz, M. (2009). Adult generation of glutamatergic olfactory bulb interneurons. Nature Neuroscience, 12, 1524-1533.

Burton, S. D. (2017). Inhibitory circuits of the mammalian main olfactory bulb. Journal of Neurophysiology, 118, 2034-2051.

de Chevigny, A., Core, N., Follert, P., Gaudin, M., Barbry, P., Beclin, C., \& Cremer, H. (2012). miR-7a regulation of Pax6 controls spatial origin of forebrain dopaminergic neurons. Nature Neuroscience, 15, 1120-1126.

Cummings, D. M., Henning, H. E., \& Brunjes, P. C. (1997). Olfactory bulb recovery after early sensory deprivation. The Journal of Neuroscience, 17, 7433-7440.

Feng, L., Zhao, T., \& Kim, J. (2015). neuTube 1.0: A new design for efficient neuron reconstruction software based on the SWC format. eNeuro, 2, 1-10.

Fiorelli, R., Azim, K., Fischer, B., \& Raineteau, O. (2015). Adding a spatial dimension to postnatal ventricular-subventricular zone neurogenesis. Development, 142, 2109-2120.

Fuentealba, L. C., Rompani, S. B., Parraguez, J. I., Obernier, K., Romero, R., Cepko, C. L., \& Alvarez-Buylla, A. (2015). Embryonic origin of postnatal neural stem cells. Cell, 161, 1644-1655.

Goebbels, S., Bormuth, I., Bode, U., Hermanson, O., Schwab, M. H., \& Nave, K. A. (2006). Genetic targeting of principal neurons in neocortex and hippocampus of NEX-Cre mice. Genesis, 44, 611-621.

Grobman, M., Dalal, T., Lavian, H., Shmuel, R., Belelovsky, K., Xu, F., ... Haddad, R. (2018). A mirror-symmetric excitatory link coordinates odor maps across olfactory bulbs and enables odor perceptual unity. Neuron, 99, 800-813.

Hayar, A., Karnup, S., Ennis, M., \& Shipley, M. T. (2004a). External tufted cells: A major excitatory element that coordinates glomerular activity. The Journal of Neuroscience, 24, 6676-6685.

Hayar, A., Karnup, S., Shipley, M. T., \& Ennis, M. (2004b). Olfactory bulb glomeruli: External tufted cells intrinsically burst at theta frequency and are entrained by patterned olfactory input. The Journal of Neuroscience, 24, 1190-1199.

Kato, Y., Kaneko, N., Sawada, M., Ito, K., Arakawa, S., Murakami, S., \& Sawamoto, K. (2012). A subtype-specific critical period for neurogenesis in the postnatal development of mouse olfactory glomeruli. PLoS One, 7, e48431.

Kay, J. N., Voinescu, P. E., Chu, M. W., \& Sanes, J. R. (2011). Neurod6 expression defines new retinal amacrine cell subtypes and regulates their fate. Nature Neuroscience, 14, 965-972.

Kosaka, T., \& Kosaka, K. (2011). "Interneurons" in the olfactory bulb revisited. Neuroscience Research, 69, 93-99.

Liu, W. L., \& Shipley, M. T. (1994). Intrabulbar associational system in the rat olfactory bulb comprises cholecystokinin-containing tufted cells that synapse onto the dendrites of GABAergic granule cells. The Journal of Comparative Neurology, 346(4), 541-558.

Liu, S., \& Shipley, M. T. (2008). Multiple conductances cooperatively regulate spontaneous bursting in mouse olfactory bulb external tufted cells. [research support, N.I.H., extramural]. The Journal of Neuroscience, 28, 1625-1639.

Madisen, L., Zwingman, T. A., Sunkin, S. M., Oh, S. W., Zariwala, H. A., Gu, H., ... Zeng, H. (2010). A robust and high-throughput Cre reporting and characterization system for the whole mouse brain. Nature Neuroscience, 13, 133-140.

Mandairon, N., Jourdan, F., \& Didier, A. (2003). Deprivation of sensory inputs to the olfactory bulb up-regulates cell death and proliferation in the subventricular zone of adult mice. Neuroscience, 119, 507-516.

Mandairon, N., Sacquet, J., Jourdan, F., \& Didier, A. (2006). Long-term fate and distribution of newborn cells in the adult mouse olfactory bulb: Influences of olfactory deprivation. Neuroscience, 141, 443-451.

Merkle, F. T., Mirzadeh, Z., \& Alvarez-Buylla, A. (2007). Mosaic organization of neural stem cells in the adult brain. Science, 317, 381-384.

Morin, X., Jaouen, F., \& Durbec, P. (2007). Control of planar divisions by the G-protein regulator LGN maintains progenitors in the chick neuroepithelium. Nature Neuroscience, 10, 1440-1448.

Najbauer, J., \& Leon, M. (1995). Olfactory experience modulated apoptosis in the developing olfactory bulb. Brain Research, 674, 245-251.
Parrish-Aungst, S., Shipley, M. T., Erdelyi, F., Szabo, G., \& Puche, A. C. (2007). Quantitative analysis of neuronal diversity in the mouse olfactory bulb. The Journal of Comparative Neurology, 501(6), 825-836.

Parrish-Aungst, S., Kiyokage, E., Szabo, G., Yanagawa, Y., Shipley, M. T., \& Puche, A. C. (2011). Sensory experience selectively regulates transmitter synthesis enzymes in interglomerular circuits. Brain Research, 1382, 70-76.

Petreanu, L., \& Alvarez-Buylla, A. (2002). Maturation and death of adultborn olfactory bulb granule neurons: Role of olfaction. The Journal of Neuroscience, 22, 6106-6113.

Qin, S., Ware, S. M., Waclaw, R. R., \& Campbell, K. (2017). Septal contributions to olfactory bulb interneuron diversity in the embryonic mouse telencephalon: Role of the homeobox gene Gsx2. Neural Development, 12, 13.

Rochefort, C., Gheusi, G., Vincent, J. D., \& Lledo, P. M. (2002). Enriched odor exposure increases the number of newborn neurons in the adult olfactory bulb and improves odor memory. The Journal of Neuroscience, 22, 2679-2689.

Roybon, L., Mastracci, T. L., Li, J., Stott, S. R., Leiter, A. B., Sussel, L., ... $\mathrm{Li}$, J. Y. (2015). The origin, development and molecular diversity of rodent olfactory bulb glutamatergic neurons distinguished by expression of transcription factor NeuroD1. PLoS One, 10, e0128035.

Sawada, M., Kaneko, N., Inada, H., Wake, H., Kato, Y., Yanagawa, Y., ... Sawamoto, K. (2011). Sensory input regulates spatial and subtypespecific patterns of neuronal turnover in the adult olfactory bulb. The Journal of Neuroscience, 31, 11587-11596.

Schindelin, J., Arganda-Carreras, I., Frise, E., Kaynig, V., Longair, M., Pietzsch, T., ... Cardona, A. (2012). Fiji: An open-source platform for biological-image analysis. Nature Methods, 9, 676-682.

Schoenfeld, T. A., \& Macrides, F. (1984). Topographic organization of connections between the main olfactory bulb and pars externa of the anterior olfactory nucleus in the hamster. The Journal of Comparative Neurology, 227, 121-135.

Schoenfeld, T. A., Marchand, J. E., \& Macrides, F. (1985). Topographic organization of tufted cell axonal projections in the hamster main olfactory bulb: An intrabulbar associational system. The Journal of Comparative Neurology, 235, 503-518.

Shih, A. Y., Mateo, C., Drew, P. J., Tsai, P. S., \& Kleinfeld, D. (2012). A polished and reinforced thinned-skull window for long-term imaging of the mouse brain. Journal of Visualized Experiments, 61, e3742.

Susaki, E. A., Tainaka, K., Perrin, D., Yukinaga, H., Kuno, A., \& Ueda, H. R. (2015). Advanced CUBIC protocols for whole-brain and whole-body clearing and imaging. Nature Protocols, 10, 1709-1727.

Tamamaki, N., Yanagawa, Y., Tomioka, R., Miyazaki, J., Obata, K., \& Kaneko, T. (2003). Green fluorescent protein expression and colocalization with calretinin, parvalbumin, and somatostatin in the GAD67-GFP knock-in mouse. The Journal of Comparative Neurology, 467, 60-79.

Tiveron, M. C., Beclin, C., Murgan, S., Wild, S., Angelova, A., Marc, J., ... Cremer, H. (2017). Zic-proteins are repressors of dopaminergic forebrain fate in mice and C. elegans. The Journal of Neuroscience, 37, 10611-10623.

Weinandy, F., Ninkovic, J., \& Gotz, M. (2011). Restrictions in time and space--new insights into generation of specific neuronal subtypes in the adult mammalian brain. The European Journal of Neuroscience, 33, 1045-1054.

Whitman, M. C., \& Greer, C. A. (2007). Adult-generated neurons exhibit diverse developmental fates. Developmental Neurobiology, 67, 1079-1093.

Whitman, M. C., \& Greer, C. A. (2009). Adult neurogenesis and the olfactory system. Progress in Neurobiology, 89, 162-175.

Winpenny, E., Lebel-Potter, M., Fernandez, M. E., Brill, M. S., Gotz, M., Guillemot, F., \& Raineteau, O. (2011). Sequential generation of olfactory bulb glutamatergic neurons by Neurog2-expressing precursor cells. Neural Development, 6, 12.

Wu, S. X., Goebbels, S., Nakamura, K., Kometani, K., Minato, N., Kaneko, T., ... Tamamaki, N. (2005). Pyramidal neurons of upper cortical layers generated by NEX-positive progenitor cells in the subventricular zone. Proceedings of the National Academy of Sciences of the United States of America, 102, 17172-17177.

Yamaguchi, M., \& Mori, K. (2005). Critical period for sensory experiencedependent survival of newly generated granule cells in the adult mouse 
olfactory bulb. Proceedings of the National Academy of Sciences of the United States of America, 102, 9697-9702.

Yang, G., Pan, F., Parkhurst, C. N., Grutzendler, J., \& Gan, W. B. (2010). Thinned-skull cranial window technique for long-term imaging of the cortex in live mice. Nature Protocols, 5, 201-208. 